\title{
YAP/TAZ regulates sprouting angiogenesis and vascular barrier maturation
}

\author{
Jongshin Kim, ${ }^{1}$ Yoo Hyung Kim, ${ }^{1,2}$ Jaeryung Kim, ${ }^{1,2}$ Do Young Park, ${ }^{1,2}$ Hosung Bae, ${ }^{1,2}$ Da-Hye Lee, ${ }^{3}$ Kyun Hoo Kim, ${ }^{1,2}$ \\ Seon Pyo Hong, ${ }^{1,2}$ Seung Pil Jang, ${ }^{1,2}$ Yoshiaki Kubota, ${ }^{4}$ Young-Guen Kwon, ${ }^{5}$ Dae-Sik Lim, ${ }^{3}$ and Gou Young Koh ${ }^{1,2}$ \\ 'Center for Vascular Research, Institute for Basic Science, Daejeon, South Korea. ${ }^{2}$ Graduate School of Medical Science and Engineering, Korea Advanced Institute of Science and Technology (KAIST), \\ Daejeon, South Korea. ${ }^{3}$ National Creative Research Initiatives Center for Cell Division and Differentiation, Department of Biological Science, KAIST, Daejeon, South Korea. ${ }^{4}$ Department of Vascular Biology, \\ The Sakaguchi Laboratory, Keio University School of Medicine, Tokyo, Japan. ${ }^{5}$ Department of Biochemistry, College of Life Science and Biotechnology, Yonsei University, Seoul, South Korea.
}

\begin{abstract}
Angiogenesis is a multistep process that requires coordinated migration, proliferation, and junction formation of vascular endothelial cells (ECs) to form new vessel branches in response to growth stimuli. Major intracellular signaling pathways that regulate angiogenesis have been well elucidated, but key transcriptional regulators that mediate these signaling pathways and control EC behaviors are only beginning to be understood. Here, we show that YAP/TAZ, a transcriptional coactivator that acts as an end effector of Hippo signaling, is critical for sprouting angiogenesis and vascular barrier formation and maturation. In mice, endothelial-specific deletion of Yap/Taz led to blunted-end, aneurysm-like tip ECs with fewer and dysmorphic filopodia at the vascular front, a hyper-pruned vascular network, reduced and disarranged distributions of tight and adherens junction proteins, disrupted barrier integrity, subsequent hemorrhage in growing retina and brain vessels, and reduced pathological choroidal neovascularization. Mechanistically, YAP/TAZ activates actin cytoskeleton remodeling, an important component of filopodia formation and junction assembly. Moreover, YAP/TAZ coordinates EC proliferation and metabolic activity by upregulating MYC signaling. Overall, these results show that YAP/TAZ plays multifaceted roles for EC behaviors, proliferation, junction assembly, and metabolism in sprouting angiogenesis and barrier formation and maturation and could be a potential therapeutic target for treating neovascular diseases.
\end{abstract}

\section{Introduction}

Formation of new blood vessels from preexisting vessels, i.e., angiogenesis, encompasses a series of morphogenic events that include sprouting at the vascular front, branching, lumen formation, anastomoses, and remodeling into a hierarchically patterned and functionally perfused vascular network $(1,2)$. Because sprouting at the vascular front is a frontline, critical process in both physiological and pathological angiogenesis, understanding the sprouting is of primary interest for controlling angiogenesis. In the vascular front, endothelial cells (ECs) are exposed to numerous angiogenic stimuli, such as growth factors including VEGF-A (hereafter VEGF) and cytokines, and adopt specific phenotypes and functions, such as tip ECs and stalk ECs, to coordinate their behaviors for regulating sprouting $(1,3-5)$. Tip ECs directionally migrate into the avascular area with extension of long, dynamic actin-based filopodia. They use filopodia to sense their surroundings for guidance cues and to steer the sprouts in a certain direction $(1,3-5)$. Trailing the tip ECs, stalk ECs proliferate to supply building blocks for growing sprouts. As the vessel elongates, stalk ECs establish tight and adherens junctions to ensure the integrity of new sprouts, create a vascular lumen, deposit a basement membrane, and associate with mural

Authorship note: Jongshin Kim and Y.H. Kim contributed equally to this work Conflict of interest: The authors have declared that no conflict of interest exists. Submitted: March 7, 2017; Accepted: June 29, 2017.

Reference information: J Clin Invest. 2017;127(9):3441-3461.

https://doi.org/10.1172/JC193825. cells $(1,3-5)$. To regulate such delicate and complex processes, several key ligands/receptors, including VEGF/VEGFR, DLL4/ Notch, angiopoietin/TIE2, Wnt/Frizzled, PDGF/PDGFR, and cell matrix/integrin, are critically and coordinately involved (1). However, key and selective transcriptional factors are beginning to be explored that mediate the signaling responses and control multiple aspects of EC behaviors for sprouting.

Yes-associated protein (YAP) or its paralog transcriptional coactivator with PDZ-binding motif (TAZ; also known as WWTR1) is a transcription coactivator that mainly interacts with the TEAD/ TEF family of transcription factors and plays crucial roles in regulating proliferation, differentiation, and migration of cells, tissue growth, and organ morphogenesis $(6,7)$. The Hippo pathway is a key upstream regulator of YAP/TAZ, limiting its activity through phosphorylation-dependent cytoplasmic retention and destabilization $(6,7)$. Interestingly, recent studies have shown that YAP/ TAZ mediates a wide range of cellular signals, including cell-cell contact, cell polarity, mechanical cues, secreted mitogens, and cellular metabolic status $(6,8-10)$, which all are also coordinately required for regulating angiogenesis. Moreover, YAP/TAZ plays pivotal roles in branching morphogenesis of lung airway and kidney ureteric duct $(11,12)$, which have structural and functional similarities with the branching morphogenesis of blood vessels during angiogenesis. Indeed, earlier studies (13-16) have revealed that core components of the Hippo pathway are involved in regulating vasculogenesis (building primitive vascular plexus with hemangioblasts) and angiogenesis during embryonic development. Previous studies $(17,18)$ have demonstrated that endothelial YAP 
activity can be modulated by an adherens junction complex, such as VE-cadherin and its adaptor EPS8, and regulates angiogenic activity of ECs. Moreover, a disturbed flow causes YAP/TAZ activation in ECs, leading to promotion of EC proliferation, inflammation, and atherogenesis $(19,20)$. Furthermore, a recent report (21) has shown that YAP can be activated by a flow-induced mechanical stress, which is independent of the Hippo pathway. However, the exact roles of YAP/TAZ and their mechanisms in angiogenesis and vascular remodeling in vivo remain unclear.

Here, we show that YAP/TAZ plays essential roles in sprouting angiogenesis and vascular barrier maturation. YAP/TAZ is critically required for migration and filopodia formation of tip ECs and proliferation of stalk ECs by regulating actin cytoskeleton remodeling and the metabolic activity of ECs. We further show that YAP/TAZ has a crucial role for maturation of the vascular barrier in retina and brain by regulating the expression and distribution of junctional and extracellular matrix (ECM) proteins and actomyosin contractility of ECs. Finally, using a mouse model of laser-induced choroidal neovascularization, we demonstrate that YAP/TAZ is also critically involved in pathological angiogenesis.

\section{Results}

Endothelial YAP/TAZ plays a promoting role in sprouting angiogenesis. To gain an insight into the role of YAP/TAZ in blood vessels, we first examined its expression in several organs in normal C57BL/6J mice. YAP was highly enriched in growing vessels of retina and brain at postnatal day 5 (P5) (Supplemental Figure 1, A and C; supplemental material available online with this article; https://doi.org/10.1172/JCI93825DS1). Notably, YAP was highly but diffusely distributed in the cytoplasm and nuclei of ECs, while TAZ was highly distributed in the nuclei of ECs, at the vascular front, and in the vascular plexus of retinal vessels (Supplemental Figure $1 \mathrm{~A}$ and Supplemental Figure 2). The publicly available microarray data (22) also revealed that YAP and its target genes are enriched in both tip and stalk ECs compared with non-ECs such as pericytes, astrocytes, and neuronal cells in retina (Supplemental Figure 1B). Further immunohistologic analysis revealed that YAP distribution was markedly reduced in the vascular plexus at P12 and in both peripheral and central regions of retinal vessels at adulthood (Supplemental Figure 2), and TAZ distribution was also markedly reduced at P12, and was barely detectable at adulthood (Supplemental Figure 2). Moreover, both YAP and TAZ were highly detectable in both cytoplasm and nuclei of ECs of cerebral cortex in brain at P5, but were reduced at P12 and further reduced at adulthood (Supplemental Figure 2). In contrast, while they were not highly detectable in ECs, they were highly and mainly distributed in nuclei of non-ECs in heart and liver at P5 and P12 (Supplemental Figure 2). These findings suggest that YAP and TAZ may play substantial roles in sprouting angiogenesis at the vascular front in retina and vessel maturation in brain in response to various stimuli during postnatal development.

Next, to investigate the roles of YAP/TAZ in growing retinal vessels, we generated a Yap/Taz ${ }^{i \Delta E C}$ mouse by crossing $\mathrm{Yap}^{f / f l} / \mathrm{Taz}^{f l / f l}$ $(23,24)$ mice with VE-cadherin-CreER ${ }^{\mathrm{T} 2}$ mice (ref. 25 and Figure $1 \mathrm{~A})$. CreER ${ }^{\mathrm{T} 2}$-positive but flox/flox-negative mice among the littermates for each experiment were defined as wild-type (WT) mice. When Yap/Taz was specifically deleted in ECs of Yap/Taz ${ }^{i \Delta E C}$ mice from P2, the mice at P5 showed delayed vessel growth and a hyper-pruned vascular network that contained fewer ECs without vascular leakage in the retina compared with those of WT mice (Figure 1, B and C). This result indicated that endothelial YAP/ TAZ contributes substantially to enhancing sprouting angiogenesis and vascular network formation in retina during the early postnatal period (birth to P5). In comparison, single deletion of Yap or Taz in growing retinal vessels using Yap ${ }^{i \Delta E C}$ mice or Taz ${ }^{i \Delta E C}$ mice showed far less abnormal vascular phenotypes compared with $\mathrm{Yap} / \mathrm{Taz}^{i \Delta E C}$ mice, indicating a certain degree of functional redundancy between YAP and TAZ in retinal vessel growth (Supplemental Figure 3).

Strikingly, the tip ECs in the vascular front region of Yap/ $T a z^{i \triangle E C}$ mice exhibited a blunted-end, aneurysm-like structure with fewer and dysmorphic filopodia compared with those of WT mice (Figure 1, D and E). The tip ECs of Yap/Taz ${ }^{i \Delta E C}$ mice had no organized F-actin bundle-containing protrusions (highlighted by phalloidin staining) and defective lumen formation (outlined by ICAM2 distribution), while those of WT mice had fine actin filament bundles protruding along filopodia and proper lumen formation (Figure 1F). These findings imply that YAP/TAZ plays crucial roles in sprouting by regulating rearrangement of cytoskeletal proteins, particularly in the tip ECs.

We confirmed markedly reduced levels of YAP and TAZ in the ECs of retinal vessels of the Yap/Taz ${ }^{i \triangle E C}$ mice (Supplemental Figure 4, A and B). Moreover, angiopoietin-2 (ANGPT2) and endothelial cell-specific molecule 1 (ESM1), which are tip EC markers as well as positive regulators of sprouting angiogenesis, but not VEGFR2, were markedly reduced in the vascular front of Yap/Taz ${ }^{i \triangle E C}$ mice compared with WT mice (Supplemental Figure 4, C-G). Furthermore, significantly fewer ETS-related genepositive $\left(\mathrm{ERG}^{+}\right) \mathrm{ECs}$ and phospho-histone $\mathrm{H} 3$-positive $\left(\mathrm{pHH}^{+}\right)$ proliferating ECs were found, but cleaved-caspase-3-positive $\left(\mathrm{cl}-\mathrm{CASP}^{+}\right)$apoptotic ECs, PDGFR $\beta^{+}$pericyte coverage onto ECs, and distribution of collagen IV-positive (COL4 ${ }^{+}$) basement membrane along ECs in the retinal vessels of $\mathrm{Yap} / \mathrm{Taz}^{i \triangle E C}$ mice were similar to those of WT mice (Figure 1, G and H, and Supplemental Figure 4, $\mathrm{H}$ and I).

Importantly, endothelial deletion of Yap/Taz from the embryo using $\mathrm{Yap}^{f / f l} \mathrm{Ta} z^{f l / l l}$ Tie2-Cre (Yap/Taz $\left.{ }^{\Delta T i e 2}\right)$ mice resulted in a severe impairment in vessel formation that led to embryonic lethality around embryonic day 9.5 (E9.5) (Supplemental Figure 5). Interestingly, YAP and TAZ were highly distributed in nuclei of ECs in dorsal skin at E14.5, which migrate toward dorsal midline (Supplemental Figure 6A). When Yap/Taz was specifically deleted in ECs of Yap/Taz ${ }^{i \triangle E C}$ mice from E9.5, the mice showed delayed capillary anastomosis with blunted vascular front and reduced branching with enlarged vessels in the dorsal skin compared with those of WT mice at E14.5 (Supplemental Figure 6, $\mathrm{B}-\mathrm{D})$. Thus, YAP/TAZ plays multiple roles in angiogenesis in an EC-autonomous manner.

Endothelial YAP/TAZ hyperactivation induces excessive filopodia and hyperplastic vascular growth. LATS1 and LATS2 kinases have a high degree of sequence homology and functional overlap and directly phosphorylate and inhibit the activities of YAP/TAZ canonically in the Hippo pathway $(6,7)$. To further elucidate the roles of 
A

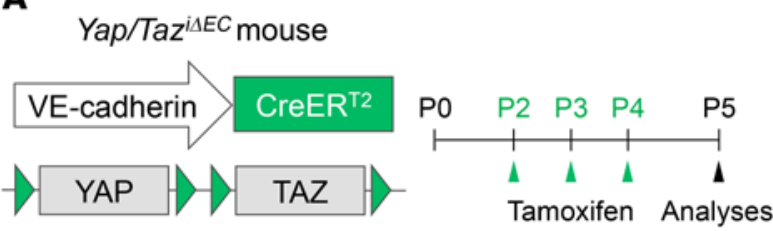

B
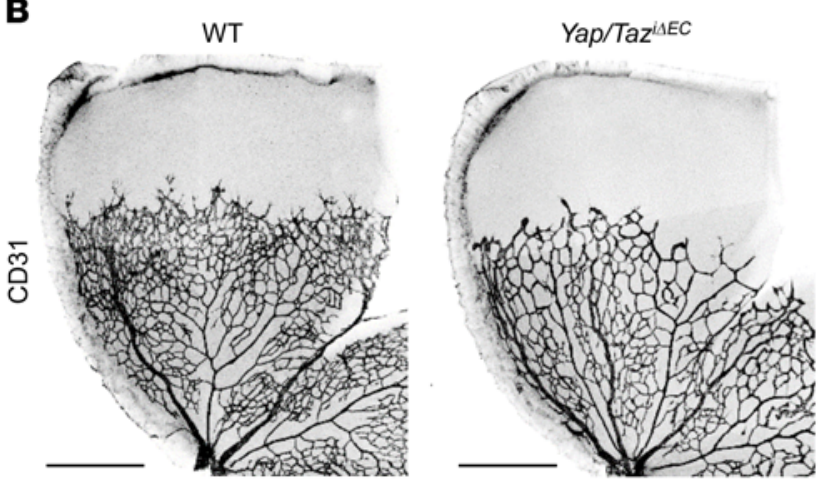

D

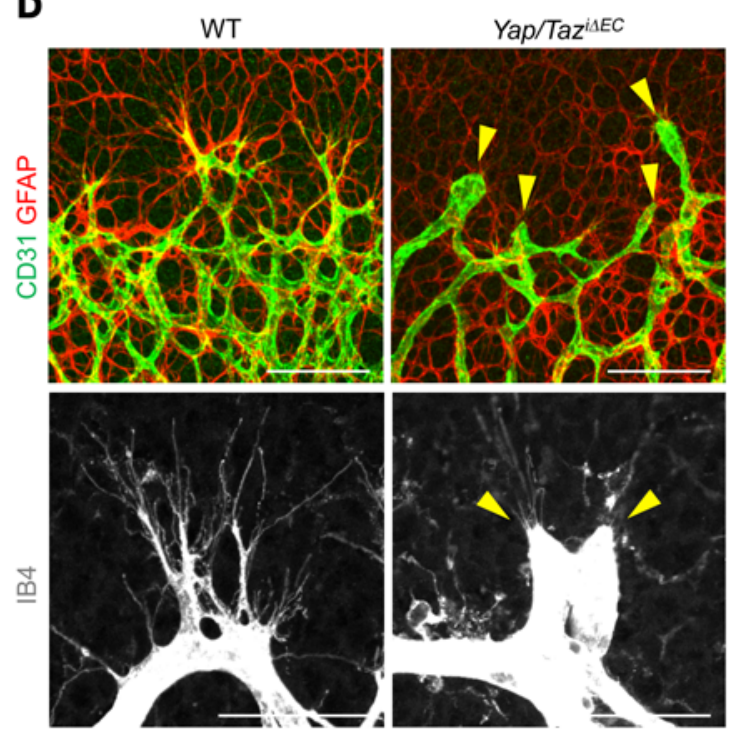

C $\quad W T=Y a p / T a z^{i \Delta E C}$
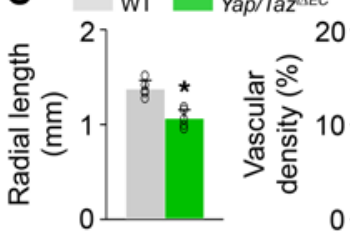

$\mathbf{F}$
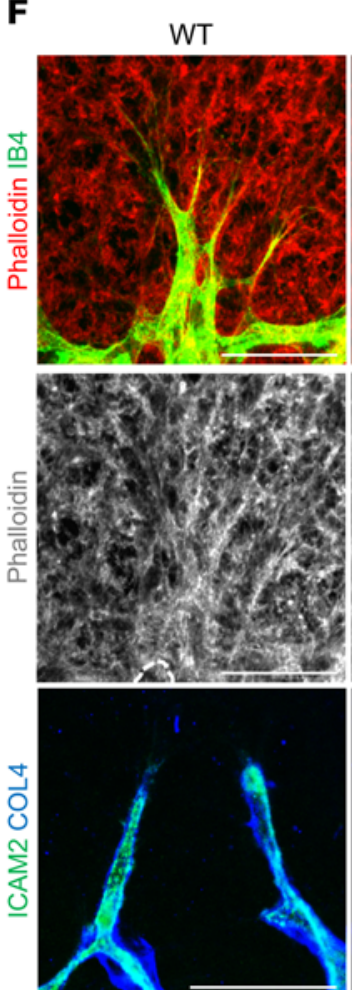

E
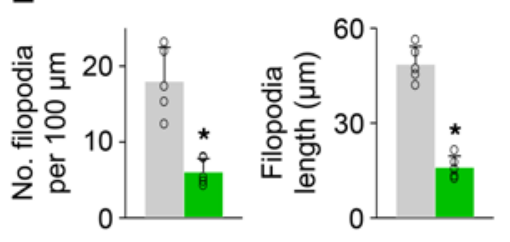

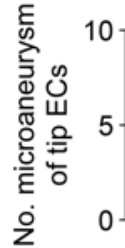

G
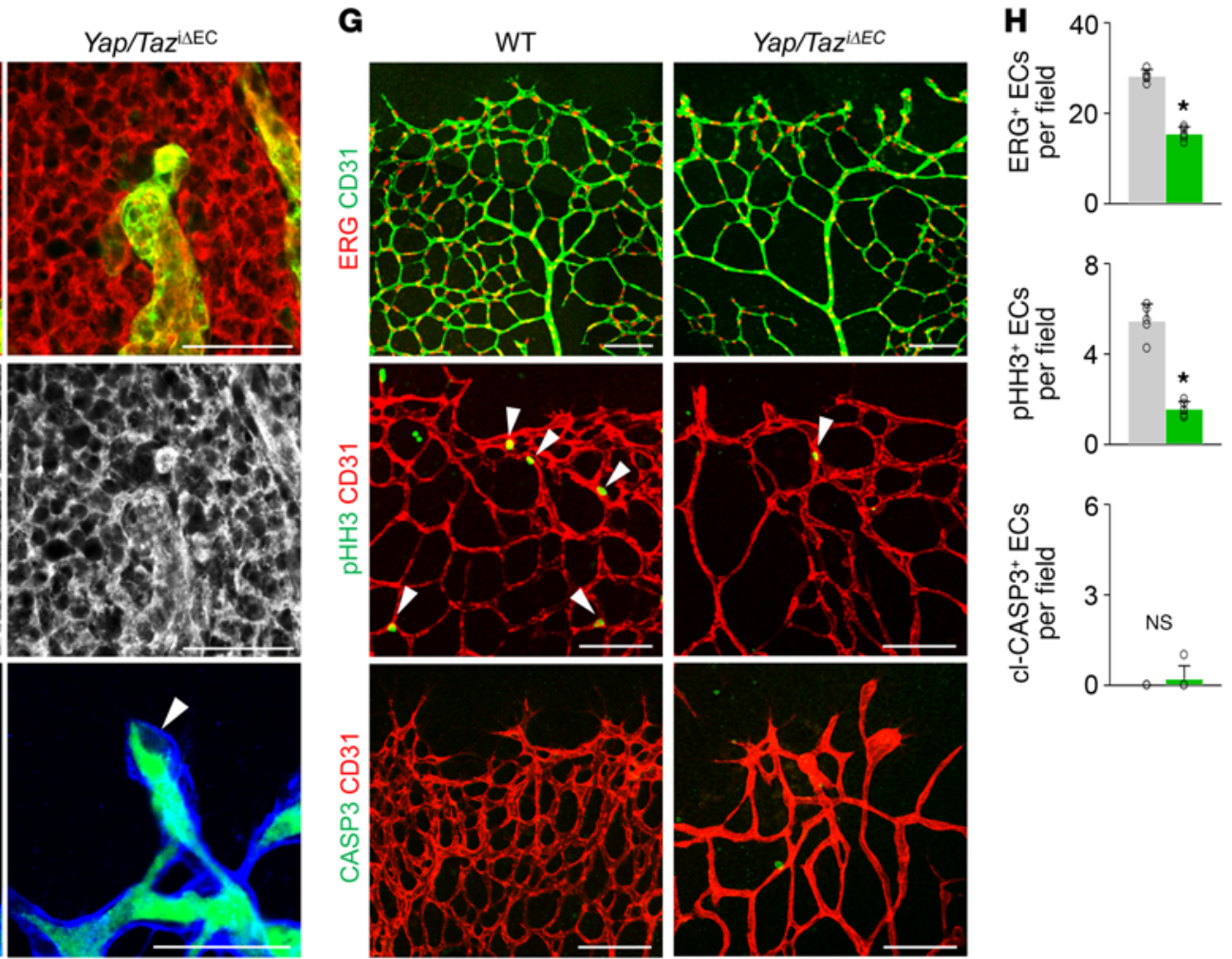

Figure 1. Endothelial YAP/TAZ is a crucial regulator of vascular sprouting and growth. (A) Diagram for EC-specific depletion of YAP/TAZ in retinal vessels from P2 and their analyses at P5 using Yap/Taz ${ }^{\text {iLCC }}$ mice. (B and C) Images of CD31+ retinal vessels and comparisons of indicated parameters in WT and Yap/Taz ${ }^{\text {AECC }}$ mice $(n=5$, each group). Scale bars: $500 \mu \mathrm{m}$. ( $\mathbf{D}$ and E) Magnified images and comparisons of retinal vessels in the vascular front region of WT and Yap/TaziliEC mice at P5 ( $n=5$, each group). Tip ECs in Yap/Taz ${ }^{i E C}$ mice exhibit an aneurysm-like structure with less and dysmorphic filopodia (yellow arrowheads). Scale bars: $100 \mu \mathrm{m}$, top panels; $50 \mu \mathrm{m}$, bottom panels. (F) Images showing phalloidin ${ }^{+}$actin filament (F-actin) bundle and ICAM2 $/$collagen $\mathrm{IV}^{+}\left(C O L 4^{+}\right)$lumen formation in tip ECs of WT and Yap/TaziAEC mice. No organized F-actin bundle-containing protrusions and defective lumen formation (white arrowhead) are detected in tip ECs of Yap/Taz ${ }^{\text {iAEC }}$ mice. Scale bars: $50 \mu \mathrm{m}$. (G and $\left.\mathbf{H}\right)$ Images and comparisons of ERG ${ }^{+}$ECs, $\mathrm{pHH}^{+}$proliferating ECs (white arrowheads), and cl-CASP3 ${ }^{+}$apoptotic ECs in WT and Yap $/$ Taz $^{\text {LEEC }}$ mice $\left(n=5\right.$, each group). Scale bars: $100 \mu \mathrm{m}$. Error bars represent mean \pm SD. ${ }^{*} P<0.05$ vs. WT by Mann-Whitney $U$ test. 
A

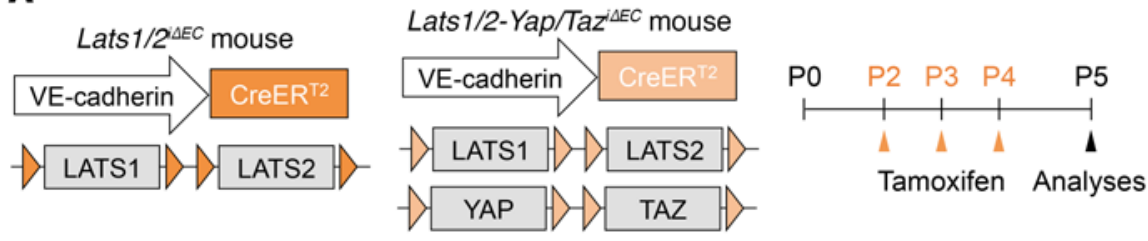

B

WT

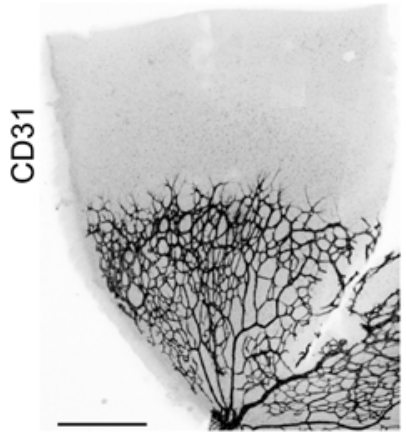

Lats $1 / 2^{\triangle A E C}$

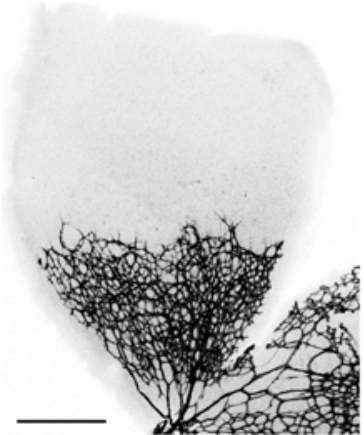

Lats 1/2-Yap/Taz ${ }^{\text {isEC }}$

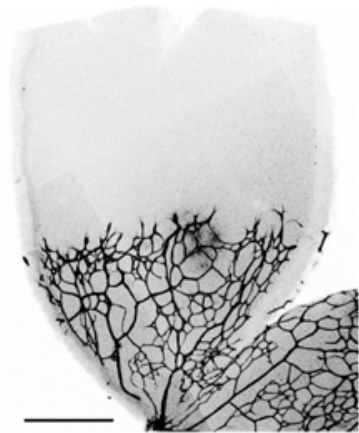

C $\quad$ Lats $1 / 2 \mathrm{WEC}_{\text {Lat }}$
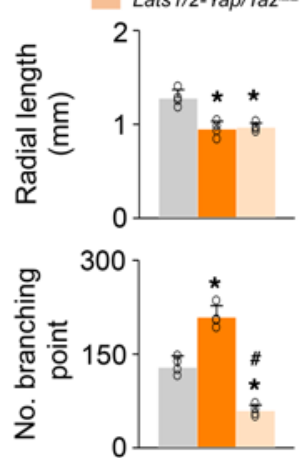

D
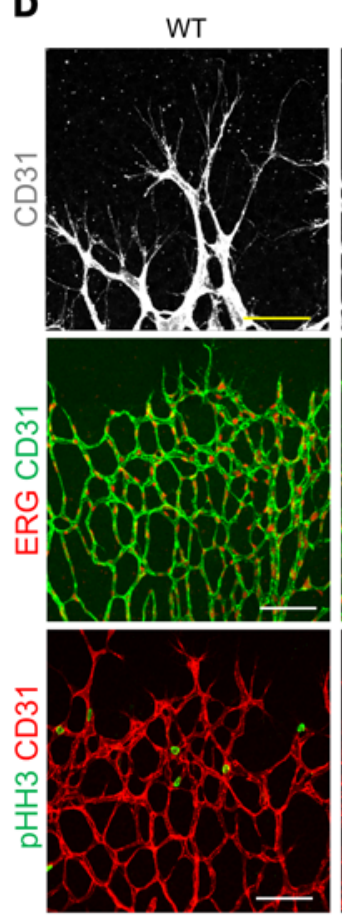

F

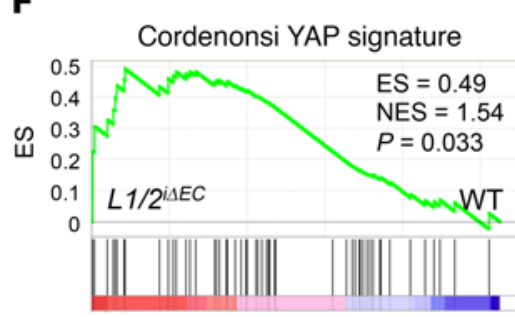

Enriched in $L 1 / 2^{i \triangle E C}$ than WT
Lats $1 / 2^{\text {iSEC }}$

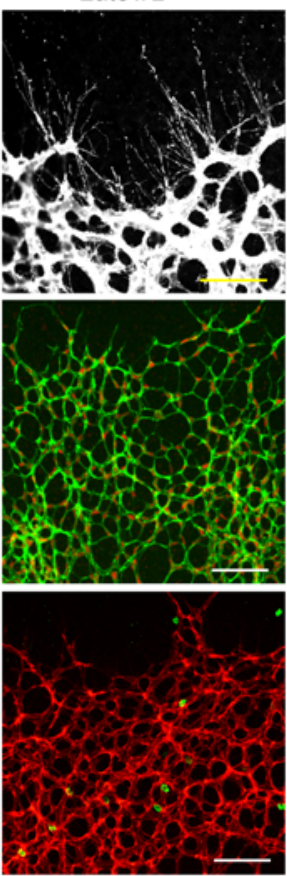

Lats 1/2-Yap/Taz ${ }^{i \Delta E C}$
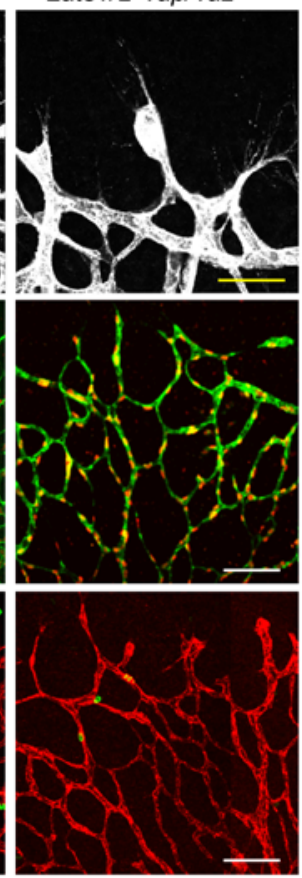

E
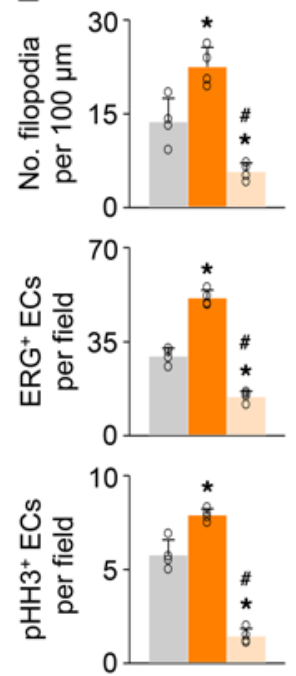

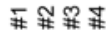

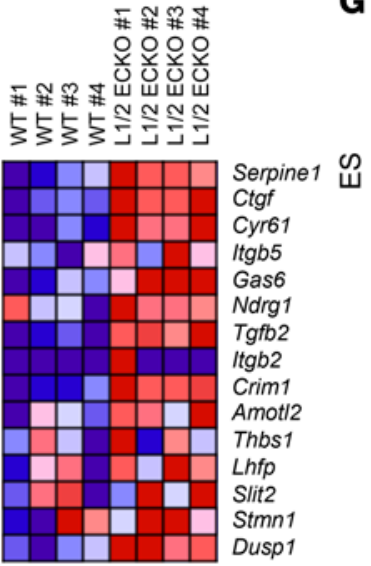

G

Cordenonsi YAP signature

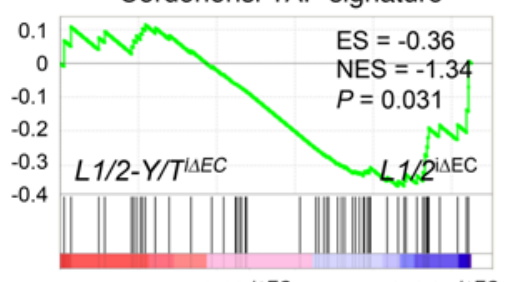

Enriched in $L 1 / 2^{i \triangle E C}$ than $L 1 / 2-Y / T^{\text {i }} E C$

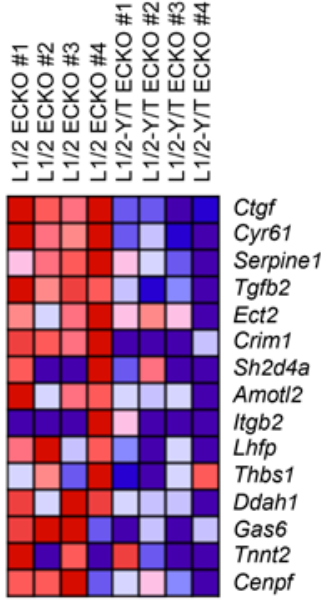


Figure 2. Endothelial Lats1/2 deletion causes dense and hyperplastic vascular network in growing retinal vessels, and their phenotypes are vanished by endothelial Yap/Taz codeletion. (A) Diagram for EC-specific deletion of Lats1/2 and double deletions of Lats $1 / 2$ and Yap/Taz in retinal vessels from P2 and their analyses at P5 in Lats1/2 ${ }^{i L E C}$ and Lats1/2-Yap/Taz ${ }^{i \leq E C}$ mice. (B and C) Images of CD31+ retinal vessels and comparisons of indicated parameters in WT, Lats1/2 $2^{\text {iAEC }}$, and Lats1/2-Yap/Taz ${ }^{i \Delta E C}$ mice ( $n=4$, each group). Scale bars: $500 \mu \mathrm{m}$. (D and $\left.\mathbf{E}\right)$ Images and comparisons of CD31+ retinal vessels in the vascular front region, ERG ${ }^{+} \mathrm{ECS}$, and $\mathrm{pHH3}^{+}$proliferative ECs in WT, Lats1/2 ${ }^{\text {iLEC }}$, and Lats1/2-Yap/Taz ${ }^{i \Delta E C}$ mice $(n=4$, each group). Scale bars: $100 \mu \mathrm{m}$. (F) CSEA of isolated lung ECs showing the upregulation of YAP signature genes in Lats $1 / 2^{\text {iLEC }}$ compared with WT mice, and corresponding heatmaps of the top 15 enriched genes. ES, enrichment score; NES, normalized enrichment score. (G) GSEA of isolated lung ECs showing the downregulation of YAP signature genes in Lats1/2-Yap/ $T a z^{i \Delta E C}$ compared with Lats $1 / 2^{i \Delta E C}$ mice, and corresponding heatmaps of the top 15 enriched genes. Error bars represent mean \pm SD. ${ }^{*} P<0.05 \mathrm{Vs}$. WT, ${ }^{\#} P<0.05$ vs. Lats $1 / 2^{\text {iAEC }}$ by Mann-Whitney $U$ test.

YAP/TAZ in vascular growth, we hyperactivated YAP/TAZ in the retinal vessels using Lats $1 / 2^{i \Delta E C}$ mice, which were generated by crossing of Lats $1^{f / f l}(26,27)$ and Lats $2^{f / f l}(28)$ mice with VE-cadherin-CreER ${ }^{\mathrm{T} 2}$ mice (Figure 2A). Lats1/2 ${ }^{i \Lambda E C}$ mice showed a dense and hyperplastic retinal vascular network but reduced radial length due to uncoordinated vascular outgrowth (Figure 2, B and C). In fact, Lats1/2 ${ }^{i \Delta E C}$ mice showed numerous filopodial bursts emerging from tip ECs at the vascular front and an increased number of ERG ${ }^{+} \mathrm{ECs}$ and $\mathrm{pHH} 3^{+}$ proliferating ECs, especially in the peripheral vascular plexus (Figure 2, D and E). Gene set enrichment analysis (GSEA) on the freshly isolated lung ECs confirmed the upregulation of YAP signature genes in Lats1/2 ${ }^{i \triangle E C}$ mice compared with WT mice (Figure $2 \mathrm{~F}$ ). Notably, these phenotypes of Lats1/2 ${ }^{i \triangle E C}$ were almost completely abolished in the retinal vessels of Lats1/2-Yap/Taz ${ }^{i \triangle E C}$ mice, which were generated by crossing of the Lats1/2 $2^{i \Delta E C}$ mice and double Yapt/fl/Taz $/ z^{f / f l}$ mice (Figure 2, D and E). YAP signature genes on the ECs were downregulated in Lats1/2-Yap/Taz ${ }^{i \Delta E C}$ compared with Lats1/2 ${ }^{i \Delta E C}$ mice (Figure $2 \mathrm{G}$ ). These findings indicate that YAP/TAZ is canonically modulated by LATS1/2 in the growing vessels and that endothelial YAP/TAZ hyperactivation induces excessive filopodia and hyperplastic vascular growth.

To address the unique roles of YAP/TAZ in tip EC formation and stalk EC proliferation, we treated $\mathrm{Yap} / \mathrm{Taz}^{i \triangle E C}$ mice at $\mathrm{P} 4$ and $\mathrm{P} 5$ with the $\gamma$-secretase inhibitor DAPT and analyzed the retinal vessels at P6 (Supplemental Figure 7A). As expected $(29,30)$, the retinal vessels of WT mice treated with DAPT showed excessive sprouting, enhanced EC proliferation, and a highly dense vascular plexus. In contrast, the unique phenotypes in the retinal vessels of Yap/ $T a z^{i \triangle E C}$ mice had not been apparently altered by DAPT treatment (Supplemental Figure 7, B and C). These findings indicate that YAP/ TAZ plays dominant roles in governing both tip EC formation and stalk EC proliferation by modulating signaling activated by multiple angiogenic cues.

Endothelial deletion of Yap/Taz leads to sustained and severe impairments in the formation and maturation of blood-retinal barrier and vision. For further determination of the role of Yap/Taz in growing retinal vessels, YAP/TAZ was deleted in the ECs from P2 in $\mathrm{Yap} / \mathrm{Taz}^{i \triangle E C}$ mice, and the retinas were analyzed at P12 (Figure $3 \mathrm{~A})$. Severe and extensive retinal and vitreous hemorrhages and profound leakage of Evans blue dye were found in the retinas of $\mathrm{Yap} / \mathrm{Taz}^{i \Delta E C}$ mice (Figure 3, B and C). Moreover, in the retinas of $\mathrm{Yap} / \mathrm{Taz}^{i \Delta E C}$ mice, there were substantially reduced vessel outgrowth and vascular density, enlarged and tortuous vessels, a relatively large avascular area at the periphery, multiple microaneurysms with no vertical sprouts in the superficial vascular plexus, and no vascular network in the deep vascular plexus (Figure 3, D-G). As a consequence, severe TER $119^{+}$rbc leakage and F4/80+ macrophage infiltration with increased plasmalemma vesicleassociated protein (PLVAP, a marker of barrier permeability; ref. 31 , reduced tight junction proteins ZO1 and claudin-5, and disarranged VE-cadherin were found in the retinas of $\mathrm{Yap} / \mathrm{Taz} \mathrm{z}^{i \Delta E C}$ mice compared with those of WT mice (Figure 3, H and I). Nevertheless, no differences were found between the 2 mouse strains in coverage of PDGFR $\beta^{+}$pericytes onto ECs in the retinal vessels (Figure 3, H and I). Importantly, these severe vascular defects and their consequences were sustained until P21 and led to severe vision impairment (Figure 4). Thus, YAP/TAZ is critically involved in formation and maturation of the blood-retinal barrier (BRB) in an EC-autonomous manner.

Endothelial YAP/TAZ is required for formation and maturation of the vascular network in the brain. Taking advantage of ECspecific deletion of Yap/Taz in a tamoxifen-inducible manner, we further evaluated whether these vascular defects occurred in other organs of $\mathrm{Yap} / \mathrm{Taz} \mathrm{z}^{\mathrm{i} E \mathrm{C} C}$ mice at P12. Although severe growth retardation and lethality were observed, most organs did not show evident vascular defects (Supplemental Figure 8, A-E). Nevertheless, weights of major organs and lengths of long bones were less in $\mathrm{Yap} / \mathrm{Taz}^{i \mathrm{iNC}}$ mice compared with those of WT mice (Supplemental Figure 8, F-I). However, the density and budding of blood vessels in the metaphysis, the number of Osterix ${ }^{+}$osteoprogenitors, and the formation of secondary ossification center in the epiphysis of $\mathrm{Yap} / \mathrm{Taz}^{\mathrm{iLEC}}$ mice were not significantly different from those of WT mice (Supplemental Figure 8, J and K). These data suggest that the growth retardation in $\mathrm{Yap} / \mathrm{Taz}^{i \triangle E C}$ mice might have resulted from malnutrition due to insufficient milk intake.

In contrast, the brains of $\mathrm{Yap} / \mathrm{Taz}^{i \triangle E C}$ mice exhibited striking, extensive, and multifocal hemorrhages, particularly in cerebral striatum, nuclei, and cerebellum at P12 (Figure 5, A-D). Further analyses revealed that substantial TER $119^{+}$rbc leakage and $\mathrm{F} 4 / 80^{+}$ macrophage infiltration together with extensive vascular leakage were detected in the multifocal regions of brains of $\mathrm{Yap} / \mathrm{Taz}{ }^{\mathrm{i} E E C}$ mice (Figure 5, E-J). Detailed histologic analyses demonstrated less vascular density with tortuous and enlarged capillaries and numerous microaneurysm formations at the vascular branching region in the brains of $\mathrm{Yap} / \mathrm{Taz}^{i \triangle E C}$ mice (Figure 6, A-C). Strikingly, the tip ECs in the vascular front region of the brain in $\mathrm{Yap} / \mathrm{Ta} z^{i \Delta E C}$ mice exhibited blunted-end, microaneurysm-like structures with almost no filopodia (Figure 6, D and E), which coincided with the phenotypes in tip ECs of the retinal vessels. Increased PLVAP and reduced transferrin receptor (TfR) but no changes in glucose transporter 1 (GLUT1) (markers for blood-brain barrier integrity; ref. 32) nor in PDGFR $\beta^{+}$pericyte coverage onto ECs were also found in the brains vessels of $\mathrm{Yap} / \mathrm{Taz}^{i \triangle E C}$ mice compared with those of WT mice (Figure 6, F and G). We confirmed efficient endothelial deletion of 

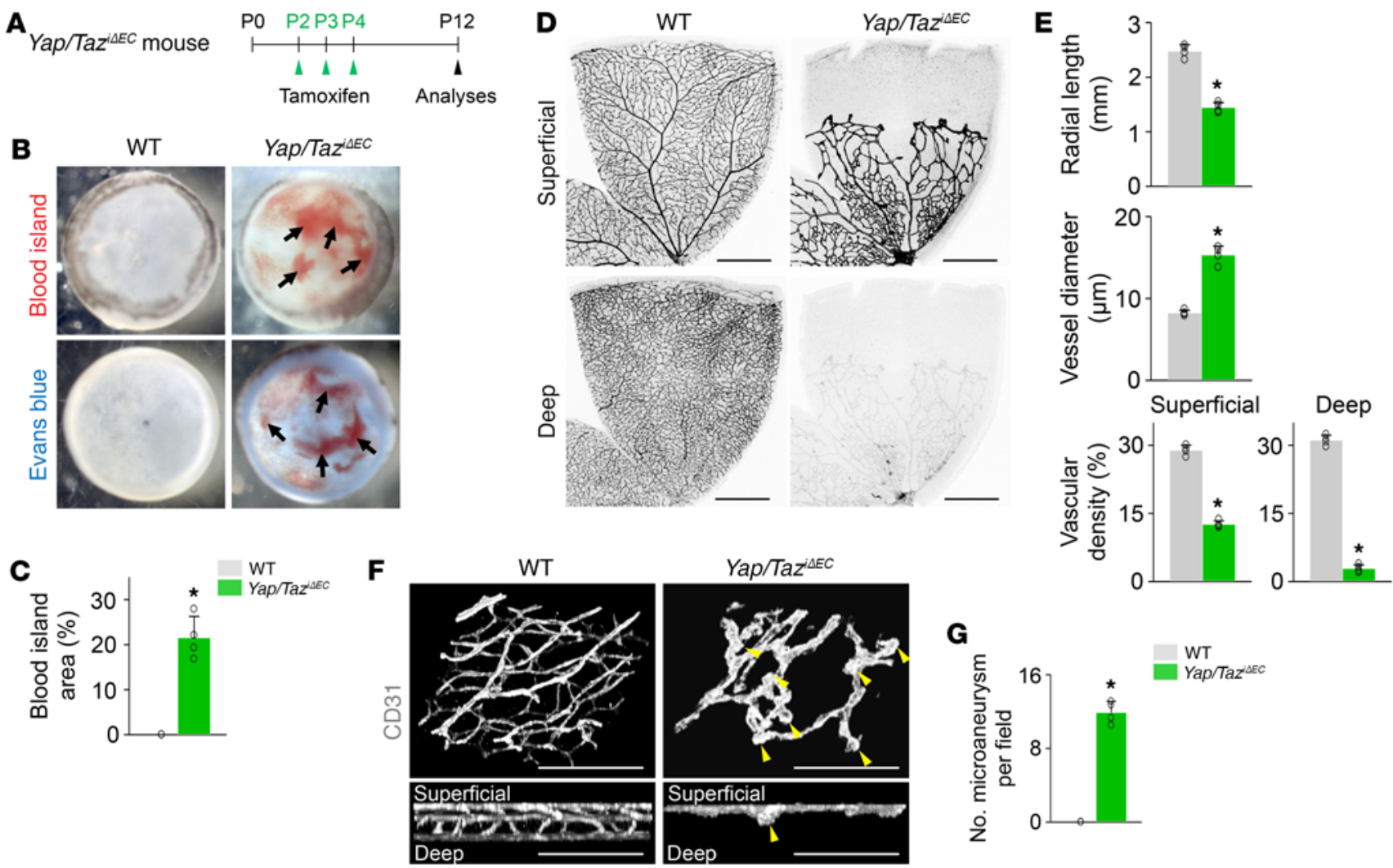

H

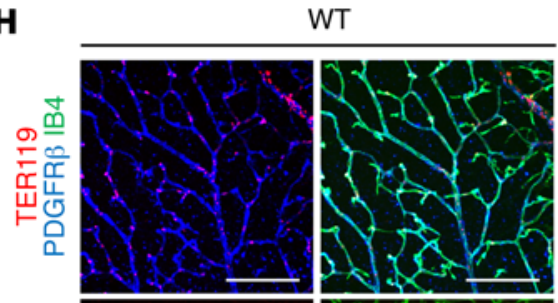

이ำ
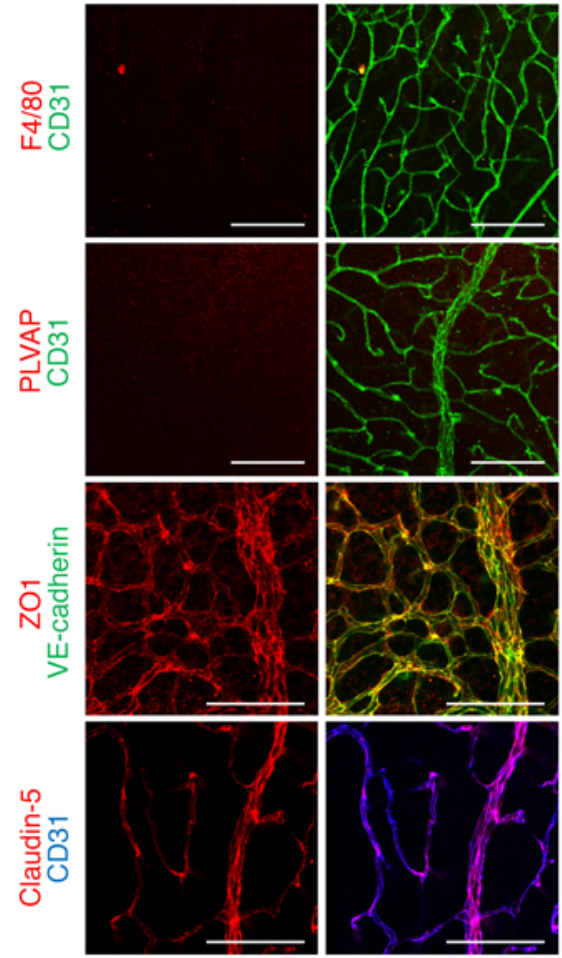

Yap/Taz ${ }^{\text {IEEC }}$
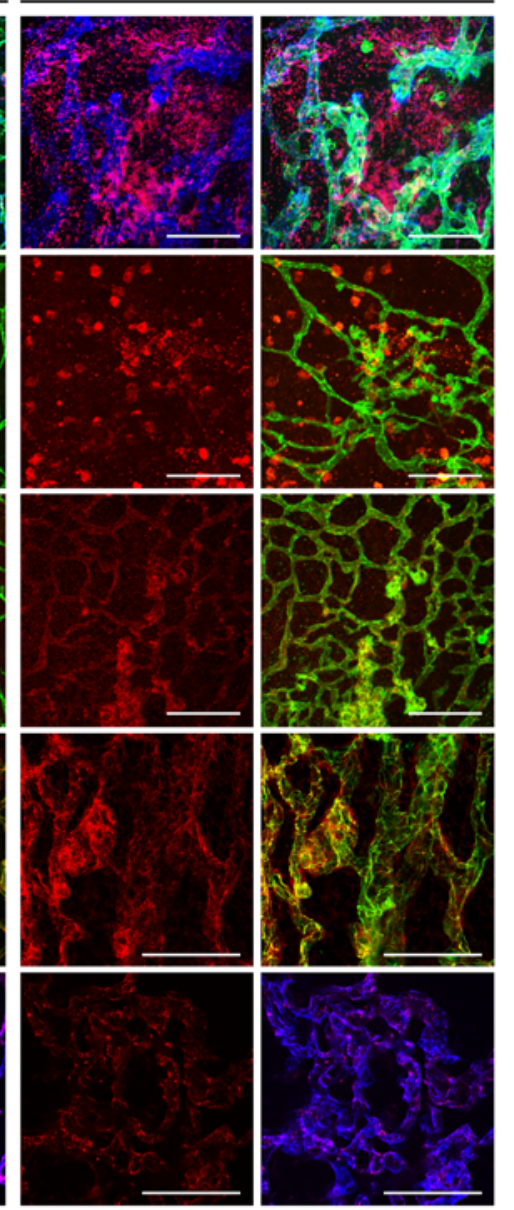
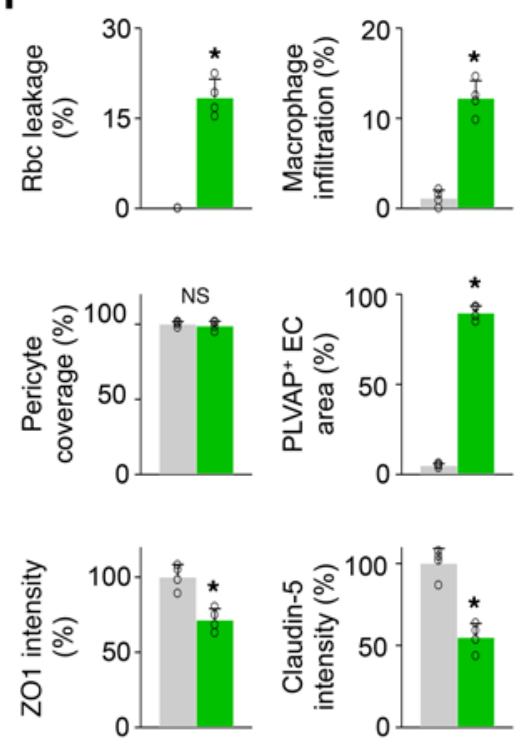
Figure 3. Endothelial Yap/Taz deletion impairs formation of vertical branching and barrier integrity in retina. (A) Diagram depicting the experiment schedule for EC-specific deletion of Yap/Taz in retinal vessels from P2 and their analyses at P12 in Yap/Taz ${ }^{i \Delta E C}$ mice. (B and C) Images of inner surface of retinal cup and comparison of blood island area of WT and Yap/TazisEc mice ( $n=4$, each group). Vitreous and retinal hemorrhage (arrows) and Evans blue (EB) leakage (bottom panels) are detected in Yap/Taz ${ }^{i L E}$ mice. (D and E) Images of CD31+ vessels in superficial and deep layers of retinas and comparisons of indicated parameters in WT and Yap/Taz ${ }^{\text {iLEC }}$ mice $(n=4$, each group). Scale bars: $500 \mu \mathrm{m}$. (F and $\mathbf{G}) 3 D$ reconstructed images of

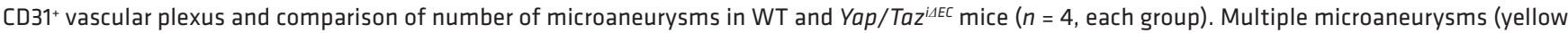
arrowheads) but no vertical branch and deep vascular plexus are observed in Yap/TazisEC mice. Scale bars: $100 \mu \mathrm{m}$. (H and I) Images and comparisons of TER $119^{+}$rbc leakage, F4/80+ macrophage infiltration, PDGFR $\beta^{+}$pericyte coverage onto CD $31^{+}$vessels, level of PLVAP on CD31+ vessels, and distributions of VE-cadherin, ZO1, and claudin-5 on CD31+ vessels in retinas of WT and Yap/Taz ${ }^{\text {iAEC }}$ mice $(n=4$, each group). Scale bars: $100 \mu \mathrm{m}$. Error bars represent mean $\pm S D$. ${ }^{*} P<0.05$ vs. WT by Mann-Whitney $U$ test.

YAP by GSEA in the isolated brain ECs and by immunohistochemistry in brain vessels of Yap/Taz ${ }^{i E C C}$ mice (Figure 6, H and I, and Supplemental Figure 8L).

It is especially notable that $\mathrm{Yap} / \mathrm{Ta} z^{i \Delta E C}$ mice had disarranged distributions and reduced levels of the tight junction proteins ZO1 and claudin-5 and the adherens junction protein VEcadherin in the ECs of brain vessels (Figure 7, A-D). Moreover, among the ECM proteins of $\mathrm{BBB}$, markedly reduced depositions of laminin and perlecan, ECM proteins of the blood-brain barrier (BBB), without changes in collagen IV and nidogen, were found along the brain capillaries in $\mathrm{Yap} / \mathrm{Taz}^{\mathrm{i} \triangle E C}$ mice compared with those of WT mice (Figure 7, E and F). mRNA expression of the genes involved in inflammation and hypoxia $(\mathrm{Ccl} 2, \mathrm{Ccl} 3$, Arg1, Tnfa, Il6, and Il10) was highly upregulated, but there were no changes in Vegf mRNA in the brains of Yap/Taz ${ }^{i \Delta E C}$ mice compared with those of WT mice (Figure 7G). Moreover, associations with the Gene Ontology (GO) terms "cell junction assembly" and "extracellular structure organization" were also reduced in brain ECs of Yap/Taz ${ }^{i \triangle E C}$ mice compared with those of WT mice (Figure 7H). Together, these results indicate that YAP/TAZ plays multifaceted roles in vascular sprouting and network formation and in BBB maturation in the brain.

YAP/TAZ controls EC migration and filopodia/lamellipodia formation by regulating actomyosin cytoskeleton. To obtain mechanical insights into how YAP/TAZ regulates sprouting angiogenesis and vascular remodeling, we performed a series of in vitro analyses in primary cultured HUVECs. Because VEGF is a key player governing sprouting angiogenesis (1-3) and recent reports $(9,33)$ demonstrate that mitogenic growth factors such as EGF activate YAP/TAZ mediated through the PI3K/PDK1/Hippo pathway, we hypothesized that VEGF might regulate YAP/TAZ activity in ECs or vice versa. Intriguingly, VEGF stimulation facilitated a nuclear localization of YAP in HUVECs (Figure 8, A and B). This finding was in accordance with the increase in VEGFR2 phosphorylation (at Tyr1175) and reductions in LATS1 phosphorylation (at Thr1079) and YAP phosphorylation (at Ser127; site that is phosphorylated by LATS1 as well as being responsible for cytoplasmic sequestration of YAP) (Figure 8, C and D). Thus, activation of VEGF/VEGFR2 signaling seems to promote the nuclear localization of YAP through the canonical Hippo pathway $(6,7)$. Moreover, mRNA levels of CTGF and CYR61, bona fide YAP target genes (34), were also increased in HUVECs after VEGF stimulation (Figure $8 \mathrm{E}$ ), consistent with previous findings (ref. 35 and Figure $8 \mathrm{~F}$ ). We then examined EC behaviors and their actin cytoskeleton using the HUVECs transfected with siControl (siCont-ECs) or siYAP/ TAZ (siYAP/TAZ-ECs). Upon VEGF stimulation, siYAP/TAZ-ECs had significantly reduced migration, smaller lamellipodia, and fewer filopodia compared with siCont-ECs (Figure 9, A-D). siYAP/ TAZ-ECs had no significant alterations in VEGF-induced phosphorylation of VEGFR2, AKT, ERK, eNOS, PAK2, or FAK compared with siCont-ECs (Figure 9E). However, among the small Rho GTPases, VEGF-stimulated enhanced CDC42 activity was markedly attenuated without changes in the activities of RhoA and Rac1 in siYAP/TAZ-ECs compared with siCont-ECs (Figure 9F). Further analysis revealed that this selective attenuation of CDC42 activity was presumably caused by substantially reduced CDC42 protein level in siYAP/TAZ-ECs (Figure 9, G and H). In addition, compared with siCont-ECs, siYAP/TAZ-ECs had markedly reduced protein level of MLC2 (also known as MYL9) without changes in the protein levels of MYH9 and MYH10 (Figure 9I). Consistent with this finding, markedly reduced pMLC2 (at Ser19) was detected in growing retinal vessels of Yap/Taz ${ }^{i \triangle E C}$ mice compared with those of WT mice (Figure 9, J and K).

Moreover, GSEA revealed that association with the GO term "regulation of actin filament based movement" was decreased in the brain ECs of $\mathrm{Yap} / \mathrm{Taz}^{\mathrm{i} E \mathrm{CC}}$ mice compared with those of WT mice (Figure 9L). These results indicate that YAP/TAZ regulates EC migration and filopodia/lamellipodia formation by modulating actomyosin cytoskeleton in response to VEGF.

YAP/TAZ regulates EC proliferation and metabolic activity through MYC signaling. Given that metabolic activity is a critical regulator for proliferation and migration of ECs during angiogenic sprouting (36-38), we then asked whether YAP/TAZ regulates metabolic activity in the HUVECs. Growth of siYAP/TAZ-ECs was markedly retarded by cell cycle arrest at $\mathrm{G}_{1}$ (Figure 10, A and B). Indeed, compared with siCont-ECs, siYAP/TAZ-ECs had marked reductions in both glycolysis and mitochondrial oxidative phosphorylation (Figure 10, C-F). Moreover, GSEA demonstrated downregulation of the genes related to E2F targets, glycolysis, and oxidative phosphorylation in the isolated brain ECs of $\mathrm{Yap} / \mathrm{Taz}{ }^{i \mathrm{iSC}}$ mice compared with WT mice (Supplemental Table 1 and Figure 10G). Notably, molecular and biochemical analyses revealed no differences in apoptosis, energy distress, senescence, or autophagy between siCont-ECs and siYAP/TAZ-ECs (Supplemental Figure 9, A-F). These results indicate that YAP/TAZ plays important roles in EC proliferation and metabolism. In particular, MYC target gene signatures were downregulated in the ECs of Yap/Taz ${ }^{i \Delta E C}$ mice compared with WT mice (Supplemental Table 1, Figure 10H, and Supplemental Figure 9G).

Because MYC is a powerful driver of glycolysis, mitochondrial metabolism, and cell growth $(39,40)$, we speculated that YAP/TAZ could modulate expression and activity of MYC in ECs. YAP/TAZ depletion suppressed protein level of MYC but 
not mRNA level of MYC in ECs (Figure 10, I and J), which is consistent with previous findings in other cells (41). Thus, these findings point to MYC as an important functional effector of YAP/TAZ in coordinating EC proliferation and metabolism during angiogenic sprouting.

Endothelial YAP/TAZ is required for pathological angiogenesis but dispensable for maintaining barrier integrity during adulthood. We finally asked whether endothelial YAP/TAZ is required for maintaining barrier integrity. To answer this question, we deleted Yap/Taz in ECs at adulthood (Figure 11A). However, Yap/Taz ${ }^{i \Delta E C}$ mice showed no definitively abnormal phenotypes, including vascular leakage, in the vessels of retina and brain (Figure 11, B-D). We also found that $\mathrm{Yap} / \mathrm{Taz}^{i \triangle E C}$ mice had similar expression and distribution of GLUT1, TfR, and PLVAP in brain vessels and PDGFR $\beta^{+}$pericyte coverage compared with WT mice (Figure 11, $\mathrm{E}$ and $\mathrm{F}$ ). These results indicate that endothelial YAP/TAZ is not required for maintaining BBB and BRB integrity in adulthood.

We next questioned whether endothelial YAP/TAZ has a substantial role in pathological angiogenesis during adulthood because higher transcriptional or metabolic activity is required to stimulate quiescent adult ECs to facilitate pathological neovascularization. To answer this question, we used a mouse model of laser-induced choroidal neovascularization (CNV) that mimics human neovascular age-related macular degeneration, one of the most common forms of ocular neovascular diseases. We specifically deleted Yap/ Taz in the ECs of blood vessels of 8-week-old Yap/Taz ${ }^{i \triangle E C}$ mice and performed laser photocoagulation 4 weeks later (Figure 11G). Yap/ $T a z^{i \triangle E C}$ mice exhibited markedly attenuated CNV volume and suppressed vascular leakage compared with WT mice at 7 days after laser photocoagulation (Figure 11, $\mathrm{H}$ and I). These findings demonstrate that YAP/TAZ is substantially involved in pathological angiogenesis during adulthood.

\section{Discussion}

In this study, we uncovered crucial roles of YAP/TAZ in morphogenesis, polarization, and migration of tip ECs and proliferation of stalk ECs in the growing vascular front by regulation of actin cytoskeleton rearrangement, cell cycle progression, and metabolism. Strikingly, the EC-specific deletion of Yap/ Taz resulted in unique morphologic alterations that comprised a nonpolarized, microaneurysm-like shape and a disorganized actin cytoskeleton with fewer and dysmorphic filopodia in the tip ECs. Consequently, sprouting angiogenesis was substantially retarded in both physiological and pathological conditions, providing evidence of the roles of YAP/TAZ as novel regulators for promoting angiogenesis.

The preferential nuclear localizations of YAP and TAZ in ECs of vascular front in growing vessels imply their substantial roles in primarily sprouting angiogenesis in response to various stimuli, but minor roles in matured and established blood vessels with little or no nuclear localizations. In fact, the impaired vascular phenotypes in Yap/Taz ${ }^{i \Delta E C}$ mice were mainly detected in the organs and at the time when nuclear localizations of YAP and TAZ were high. A recent report (21) showed that the blood flow-dependent YAP nuclear localization in ECs is independent of the Hippo pathway. On the other hand, we and others (17) have observed nuclear localization of TAZ in the vascular front of the retina, where flow is absent but a strong VEGF gradient toward the front is present. In support of this observation, our biochemical analyses in primary cultured HUVECs revealed that activation of VEGF/VEGFR2 signaling appears to promote nuclear localization of YAP through the canonical Hippo pathway $(6,7)$. Therefore, VEGF may be one of the major blood flow-independent stimuli that activate YAP/TAZ in ECs in the vascular front area of growing vessels, which promotes sprouting angiogenesis.

We note that the unique vascular phenotypes in $\mathrm{Yap} / \mathrm{Taz} \mathrm{z}^{i \Delta E C}$ mice are very similar to those of $S r f^{\triangle E C}$ and $M r t f^{\triangle E C}$ mice (42-44), which have an EC-specific deletion of serum response factor ( $\mathrm{Srf}$ ) or its transcriptional cofactor myocardin-related transcriptional factor (Mrtf). Like the alterations in retinal vessels of $\mathrm{Yap} / \mathrm{Taz}^{i \Delta E C}$ mice, $S r f^{\triangle \triangle E C}$ and $M r t f^{\triangle \triangle E C}$ mice exhibit blunted-end, aneurysm-like structures with impaired filopodia protrusion of tip ECs, incomplete formation of the primary vascular plexus, and an absence of the deep plexus. Interestingly, both MRTF and YAP/TAZ use substantially overlapping regulation mechanisms in response to mechanical signals $(10,45)$. Moreover, there are multiple levels of crosstalk between the MRTF-SRF and YAP/TAZ-TEAD cascades (46-50). In fact, both MRTF-SRF and YAP/TAZ signature genes substantially overlap, and hundreds of these genes are critically involved in actin cytoskeleton-mediated processes such as cell motility, actin dynamics, cell adhesion, and ECM synthesis (46). Therefore, it is tempting to speculate that YAP/TAZ-TEAD and MRTF-SRF may act together to coordinate transcriptional responses to various cellular stimuli, particularly for sprouting angiogenesis. Indeed, our preliminary GSEA revealed that the MRTF signature genes are significantly downregulated in the brain ECs isolated from Yap/Taz ${ }^{i \Delta E C}$ mice (data not shown), emphasizing a potential molecular interaction between YAP/TAZ and MRTF-SRF in the ECs of growing vessels. Nevertheless, unlike the phenotype of $\operatorname{Srf}{ }^{\triangle E C}$ mice $(42,44)$, a proliferation activity of the stalk ECs is markedly reduced in the retinal vessels of $\mathrm{Yap} / \mathrm{Taz}{ }^{i \Delta E C}$ mice. ANGPT2 and ESM1 are highly enriched in the tip ECs (51), and their expressions are regulated by $\operatorname{FOXO} 1(52,53)$, while the former is regulated by YAP (17). However, the phenotypes of growing retinal vessels of $A n g p t 2^{i \triangle E C}$ (54) and $E s m 1^{i \triangle E C}$ (55) mice are different and pathologically milder than those of $Y a p / \operatorname{Taz}^{i \Delta E C}$ mice. These similarities and dissimilarities imply that YAP/TAZ has multifaceted roles in ECs for sprouting angiogenesis.

Mechanistically, we have identified potential actomyosin machineries regulated by YAP/TAZ in the ECs stimulated with VEGF. Small Rho GTPases are master regulators of the structure and dynamics of actomyosin cytoskeleton required for cell migration, polarization, adhesion, vesicle trafficking, and cell cycle $(56,57)$. Among the molecules, CDC42 is critically required for filopodia formation in ECs of angiogenic sprouts (58-61). Intriguingly, our results revealed that YAP/TAZ depletion markedly reduces Cdc42 activity in the ECs stimulated with VEGF, which could be an underlying mechanism for the impaired shape of tip ECs in Yap/Taz ${ }^{i \triangle E C}$ mice. Moreover, YAP/TAZ promotes the expression of MLC2, a component of nonmuscle myosin II that plays central roles in cell migration, adhesion, and tissue morphogenesis (62). Interestingly, these cytoskeletal regulators are also regulated by MRTF/SRF signaling (42). 
A

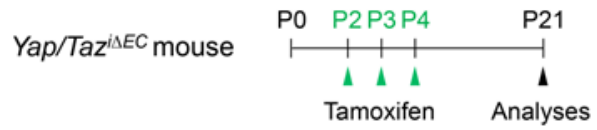

B
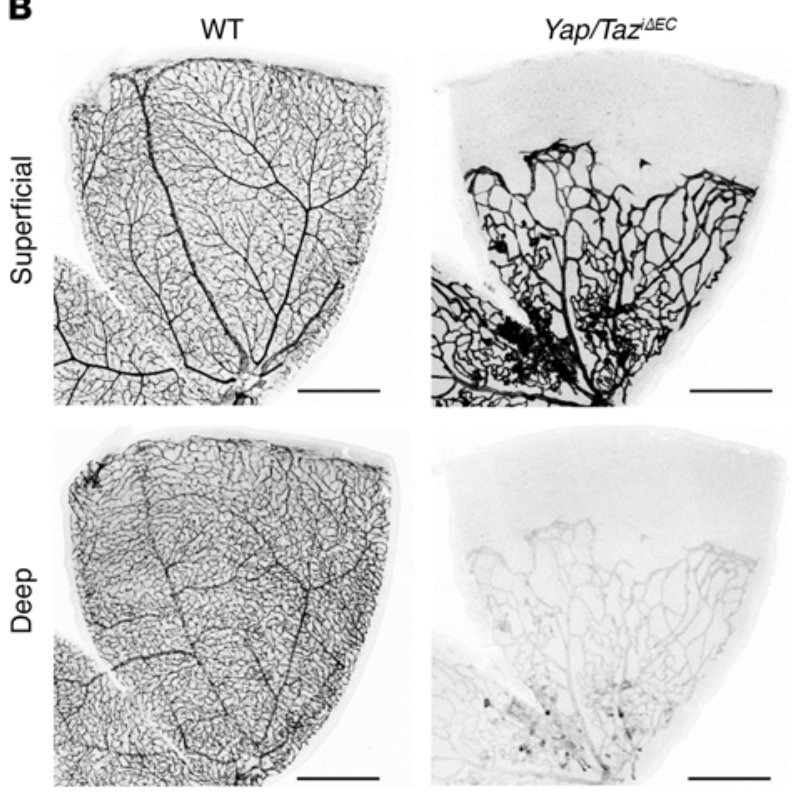

$\mathbf{E}$
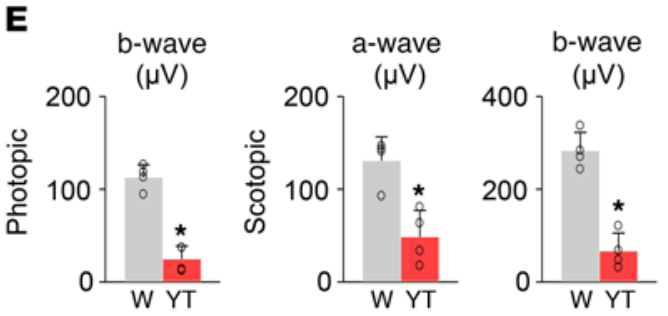

C

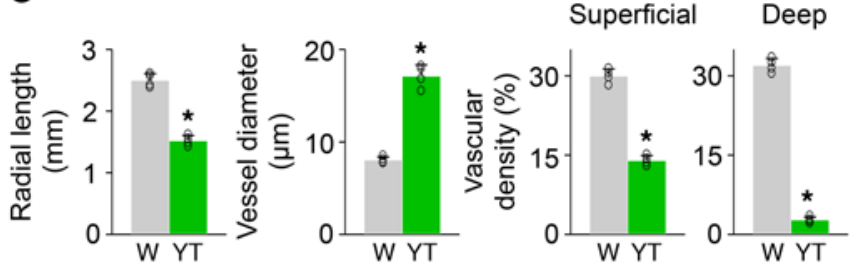

D Photopic

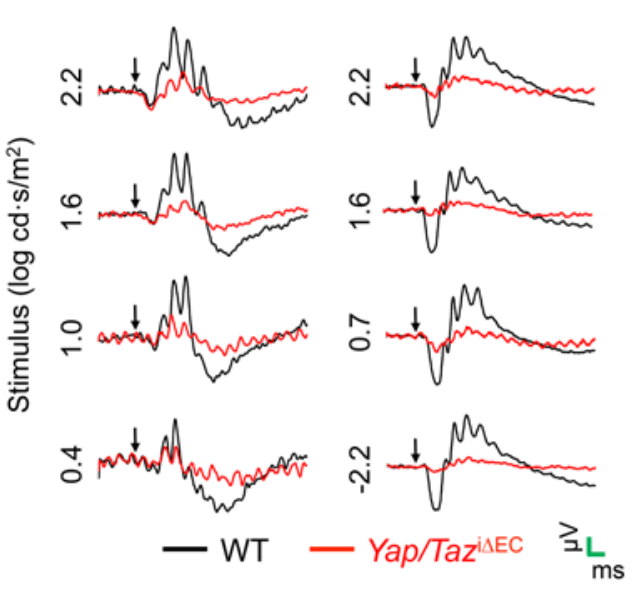

Figure 4. Endothelial Yap/Taz deletion leads to sustained and severe impairment of BRB and vision. (A) Diagram depicting the experiment schedule for EC-specific deletion of Yap/Taz in retinal vessels from P2 and their analyses at P21 in Yap/Taz ${ }^{i \Delta E C}$ mice. (B and C) Images of CD31+ vessels in superficial and deep layers of retinas and comparisons of indicated parameters in WT (W) and Yap/Taz ${ }^{\text {iAEC }}$ (YT) mice $(n=4$, each group). Scale bars: $500 \mu \mathrm{m}$. (D) Electroretinography in WT and Yap/Taz ${ }^{i \Delta E C}$ mice. Normal electroretinographic responses as shown by photopic B wave and scotopic a- and b- waves in WT mice (black line) are hardly detected in Yap/Taz ${ }^{i \leq E C}$ mice (red line). Arrows, flash stimuli. Scale bars (green): $40 \mathrm{~ms}(x)$ and $40 \mu \mathrm{V}(y)$ for photopic electroretinography; $40 \mathrm{~ms}(x)$ and $160 \mu \mathrm{V}(y)$ for scotopic electroretinography. (E) Comparisons of amplitude of photopic b-wave and scotopic a- and b- waves in WT and Yap/Taz ${ }^{i A E C}$ mice $\left(n=4\right.$, each group). Error bars represent mean \pm SD. ${ }^{*} P<0.05$ vs. WT by Mann-Whitney $U$ test.

Moreover, a recent study (63) has shown that YAP is indispensable for tissue morphogenesis mediated through a Rho GTPase-activating protein, ARHGAP18, to regulate the cortical actomyosin network and filopodia formation in medaka and zebrafish. Together, our findings reinforce the idea that actomyosin rearrangement is an essential mediator of YAP/TAZ for directional migration and filopodia formation of tip ECs during sprouting in response to angiogenic cues (Figure 12). Further detailed study is required to ascertain the YAP/TAZ-induced regulations of CDC42 and MLC2 and consequent changes in filopodia formation and junction assembly in growing vessels.

Emerging results (36-38) have highlighted the importance of EC metabolism in controlling the behaviors of tip and stalk ECs during angiogenic sprouting. VEGF enhances glycolysis in ECs by inducing expression of GLUT1 and glycolytic enzymes such as PFKFB3 and LDHA, and this VEGF-enhanced glycol- ysis promotes tip EC migration and stalk EC proliferation in the angiogenic front (64). As a downstream metabolic effector, MYC is highly enriched in the ECs of sprouting vessels, and its depletion reduces both glycolysis and mitochondrial respiration in ECs (40). Moreover, $M y{ }^{i \Delta E C}$ mice show impaired vascular growth and branching (40), similar to the vascular phenotypes of Yap/Taz ${ }^{i \Delta E C}$ mice. A recent study (65) indicates that YAP promotes cell proliferation and glycolysis by upregulating GLUT3 expression in transformed cell lines. In accordance with these studies, our findings indicate that YAP/TAZ promotes not only EC proliferation and metabolism but also the expression and activity of MYC in the ECs. In this regard, YAP/TAZ may regulate proliferation and metabolism through MYC signaling in stalk ECs during angiogenic sprouting (Figure 12). However, fatty acid oxidation (FAO) is specifically required for EC proliferation during angiogenic sprouting (66), and YAP promotes 
A

Yap/Taz' ${ }^{i \text { EEC }}$ mouse
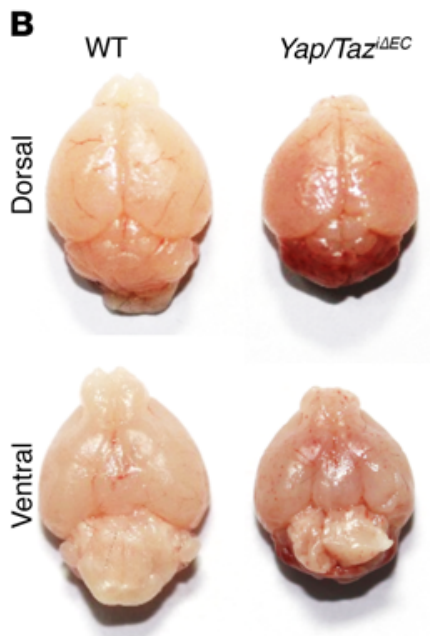

E

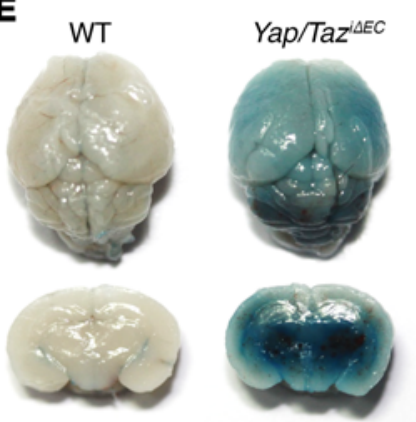

Evans blue

G
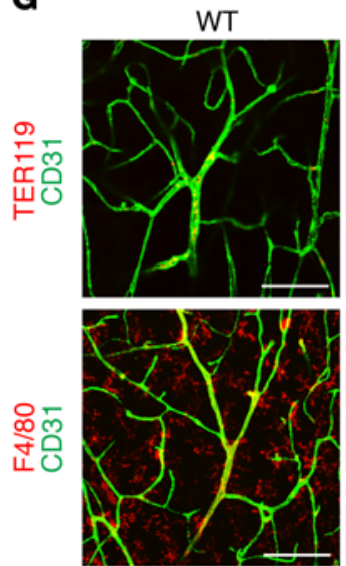

C
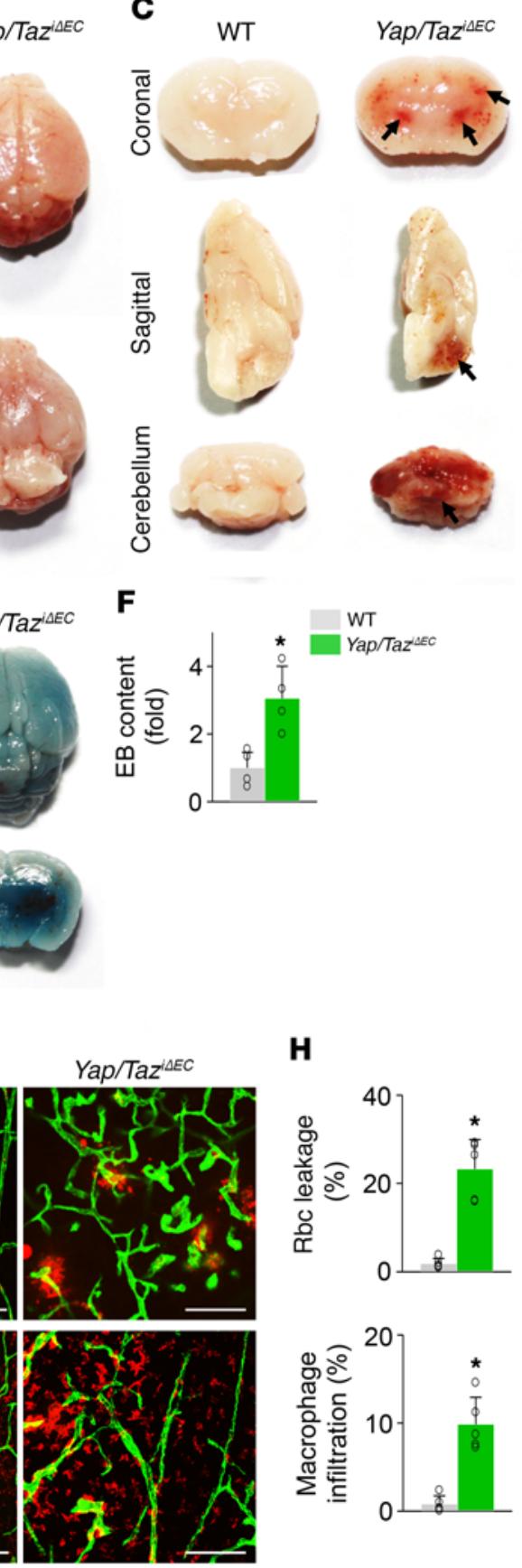

H
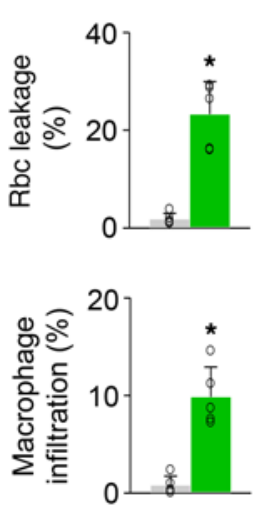

D

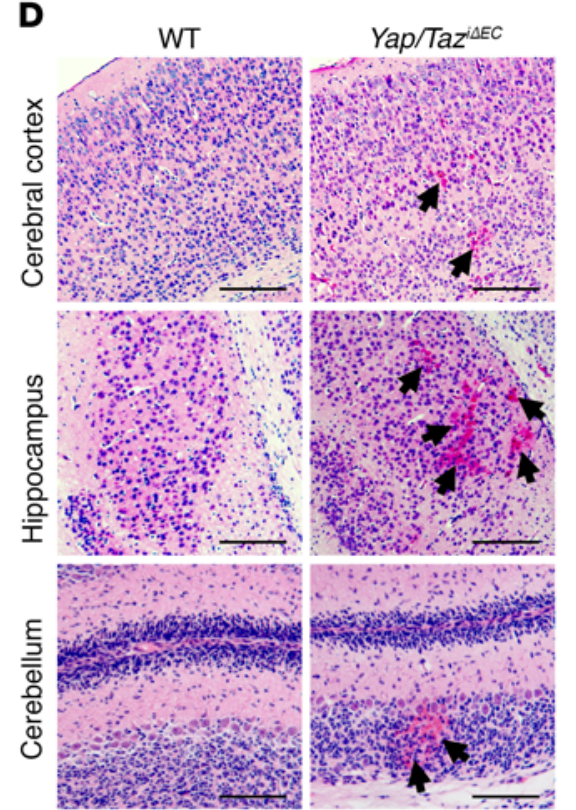

I
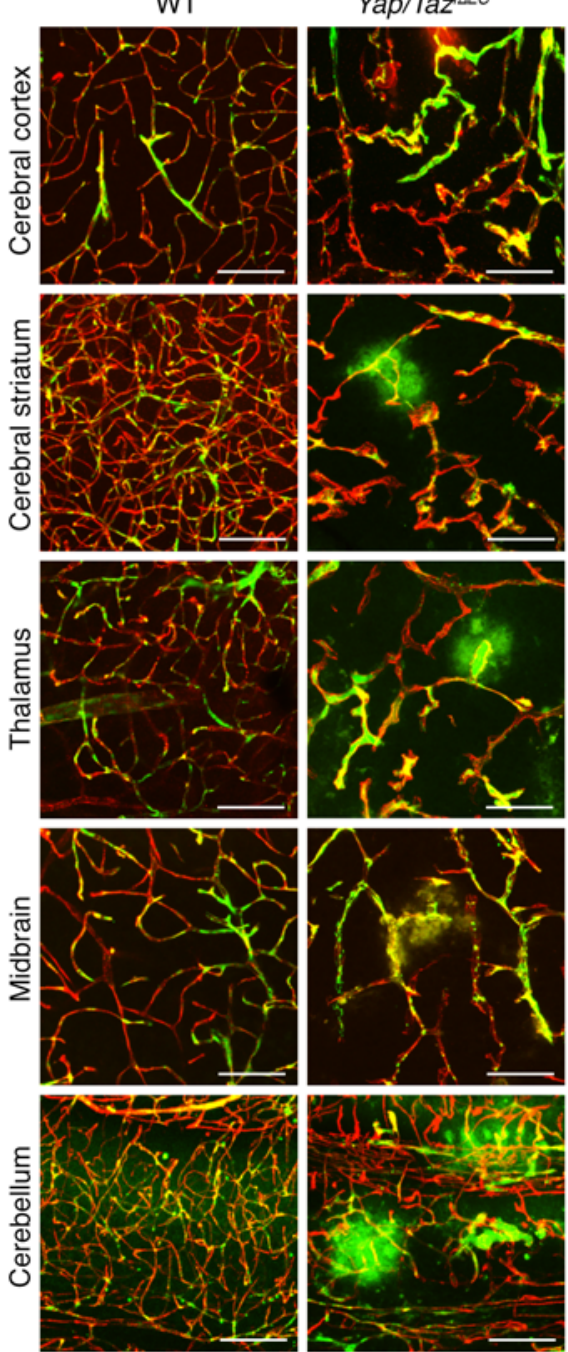

Dextran CD31
J
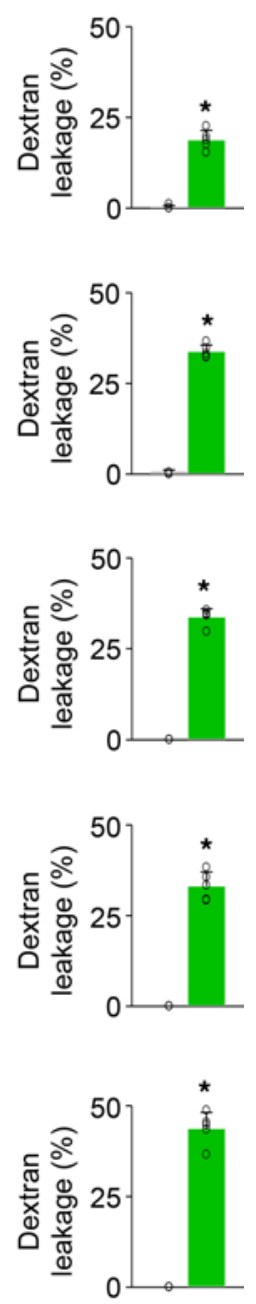
Figure 5. Endothelial Yap/Taz deletion leads to extensive and multifocal brain hemorrhage. (A) Diagram depicting the experiment schedule for Yap/ Taz deletion in brain ECs from P2 and their analyses at P12 in Yap/Taz ${ }^{i \Delta E C}$ mice. (B and C) Images of whole and sectioned brains. Severe hemorrhage is detected in cerebral striatum and nuclei, and cerebellum (arrows). (D) H\&E staining of brain sections at indicated areas. Brain hemorrhage (arrows) is detected in Yap/TaziLEC mice. Scale bars: $100 \mu \mathrm{m}$. (E and F) Images of whole and sectioned brains and comparison of Evans blue (EB) leakage in brains after EB injection in WT and $\mathrm{Yap} / \mathrm{Taz}^{\mathrm{i}}{ }^{\mathrm{AEC}}$ mice ( $n=4$, each group). ( $\mathbf{G}$ and $\mathbf{H}$ ) Images and comparisons of TER119+ rbc leakage and F4/80+ macrophage infiltration in cerebral striatum of WT and Yap/TaziAEC mice ( $n=5$, each group). Scale bars: $100 \mu \mathrm{m}$. (I and J) Images and comparisons of FITCconjugated dextran $(70 \mathrm{kDa}$ ) leakage in the indicated areas of WT and Yap/ Taz ${ }^{i \Delta E C}$ brains ( $n=5$, each group). Scale bars: $100 \mu \mathrm{m}$. Error bars represent mean \pm SD. ${ }^{*} P<0.05$ vs. WT by Mann-Whitney $U$ test.

expression of CPT1A, a rate-limiting enzyme of FAO in ECs (67). Thus, it would be worth examining whether YAP/TAZ controls FAO activity in ECs.

This study also demonstrates that YAP/TAZ is critically required for formation and maturation of the $\mathrm{BRB}$ and $\mathrm{BBB}$. Building of the $\mathrm{BRB}$ and $\mathrm{BBB}$ begins with coordinated induction of proteins that maintain tight and adherens junctions and nutrient transporters on the ECs at the onset of vascular branching and network formation, followed by maturation of the barrier properties along with close interactions with pericytes and Müller cells, glial cells, or astrocytes in retina and brain (68-70). Notably, the assembly and maturation of junctional complexes are intimately linked to the actomyosin dynamics and the activity of small Rho GTPases such as CDC42 $(61,71)$, which can be regulated by YAP/ TAZ. Thus, the impairments in the BRB and BBB in Yap/Taz ${ }^{i \triangle E C}$ mice appear to be caused by multiple defects in these sequential processes, implying that $\mathrm{YAP} / \mathrm{TAZ}$ as a transcriptional coactivator plays multiple roles in differentiation and maturation of the growing vessels. YAP/TAZ is dispensable for maintaining blood-barrier integrity during adulthood, but it contributes to inducing pathological angiogenesis. Thus, YAP/TAZ plays dynamic roles in the ECs of growing vessels, which require high levels of cell dynamic transcription or metabolism.

Together, the findings of this study delineate the essential roles of YAP/TAZ for sprouting angiogenesis and barrier maturation and identify their multifaceted actions as core transcriptional regulators mediating angiogenic signals and navigating EC behaviors. These behaviors include directional migration, proliferation and metabolism, and junction assembly, illuminating the complexity and delicate control of angiogenesis. Our results also provide a basis for targeting vascular sprouting as a potential therapeutic strategy for pathological angiogenesis.

\section{Methods}

Supplemental Methods are available online with this article; https:// doi.org/10.1172/JCI93825DS1.

Mice and treatment. Specific pathogen-free (SPF) C57BL/6J mice and Tie2-Cre mice were purchased from the Jackson Labo-

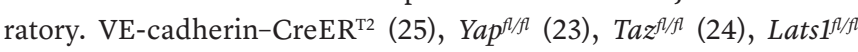
$(26,27)$, and Lats $2^{f l / f l}$ (33) mice were transferred, established, and bred in our SPF animal facilities at KAIST and fed with free access to a standard diet (PMI LabDiet) and water. In order to induce Cre activity in the CreER ${ }^{\mathrm{T} 2}$ mice, tamoxifen was given with the following dosages and schedules: for embryos, $2 \mathrm{mg}$ of tamoxifen (SigmaAldrich, T5648) dissolved in corn oil (Sigma-Aldrich, C8267) was injected i.p. into the pregnant mother at E9.5 and E11.5; for neonatal mice, $100 \mu \mathrm{g}$ of tamoxifen was injected either into the stomach or subcutaneously every day from P2 to P4; for adult mice aged over 1 month, $2 \mathrm{mg}$ of tamoxifen was injected i.p. over 5 consecutive days from the indicated time point. For constitutive Cre recombination in the Cre mice, Cre-positive male mice and Cre-negative female mice were used for intercrossing and embryos were obtained at E9.5. For in vivo inhibition of DLL4/Notch signaling, $100 \mathrm{mg} / \mathrm{kg}$ of $\gamma$-secretase inhibitor (DAPT; $N-[N-(3,5-$ difluorophenacetly)-L-alanyl]-S-phenylglycine t-butyl ester) (Calbiochem, 565770) dissolved in corn oil was injected subcutaneously at P5 and P6 after tamoxifen administration, and the retinas were harvested at P7. For anesthesia, mice were injected i.p. with the anesthetic solution (ketamine $40 \mathrm{mg} / \mathrm{kg}$ and xylazine $5 \mathrm{mg} / \mathrm{kg}$ ).

Laser-induced CNV mouse model. Generation of the laser-induced CNV mouse model was performed using approximately 8-week-old male mice according to a previously described method (72) with some modifications (73). After dilation of pupils with $1 \%$ tropicamide and 2.5\% phenylephrine eye drops (Santen Pharmaceutical) and instillation of $0.5 \%$ proparacaine hydrochloride eye drops (Alcon) for topical anesthesia, a laser photocoagulator (Lightmed, LIGHTLas 532) with a slit lamp delivery system was used with a glass coverslip as a contact lens to visualize the retina. Sufficient laser energy $(532 \mathrm{~nm}$ wavelength, $250 \mathrm{~mW}$ power, $100 \mathrm{~ms}$ duration, $50 \mu \mathrm{m}$ spot size) was delivered in 4 locations for each eye (the 3, 6, 9, and 12 o'clock positions of the posterior pole). Only burns that produced a bubble at the time of laser photocoagulation, indicating the rupture of the Bruch membrane, were included in this study. Spots containing hemorrhage at the laser site were excluded from the analysis.

Vascular permeability assay. For dextran leakage assay, FITCconjugated dextran (70 kDa; Sigma-Aldrich, 46945) was injected at a concentration of $25 \mathrm{mg} / \mathrm{ml}$ into the retro-orbital plexus $30 \mathrm{~min}$ utes before harvesting of brains. For Evans blue (EB) leakage assay, $100 \mu \mathrm{l}$ of EB (2\% in PBS; Sigma-Aldrich, E2129) was injected i.p. 24 hours before harvesting of brains. To quantify EB leakage in brains, harvested brains were homogenized and incubated in formamide for 24 hours at $55^{\circ} \mathrm{C}$. Supernatants were collected and EB was measured at the absorbance of $620 \mathrm{~nm}$. EB concentrations were normalized by control, calculated from a standard curve of EB in formamide, and presented as fold difference.

Confocal scanning laser fluorescein angiography and indocyanine green angiography. Using a custom-built confocal scanning laser retinal angiography system (74), in vivo fundus fluorescein angiography (FA) and indocyanine green angiography (ICGA) of the CNV lesion were performed. Continuous-wave laser modules at $488 \mathrm{~nm}$ and 785 $\mathrm{nm}$ were used as excitation sources for fluorescein and indocyanine green, respectively. A raster scanning pattern of excitation lasers was achieved by a scanner system consisting of a rotating polygonal mirror (Lincoln Laser, MC-5) and a galvanometer-based scanning mirror (Cambridge Technology, 6230H), and delivered to the back aperture of an imaging lens. A high numerical aperture (NA) objective lens (PlanApo $\lambda$, NA 0.75; Nikon) was used as the imaging lens to provide wide-field fundus fluorescence images. Fluorescence signals detected by photomultiplier tubes (Hamamatsu Photonics, R9110) were digi- 
A

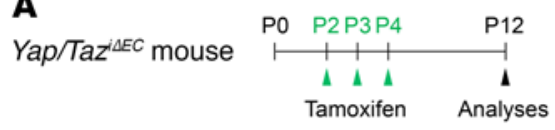

B

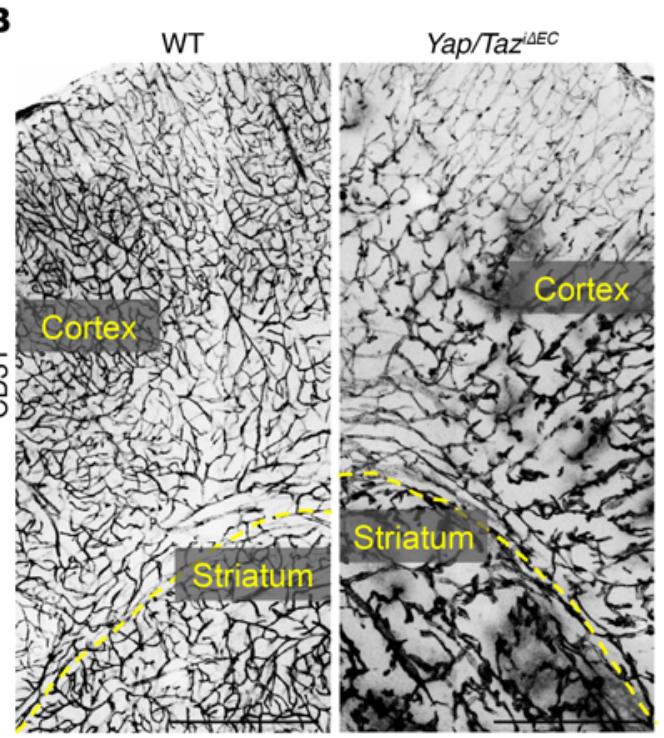

F

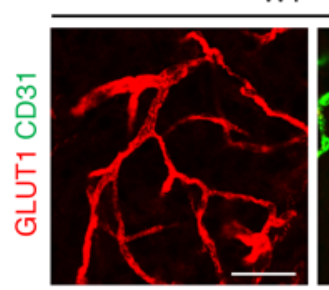

WT
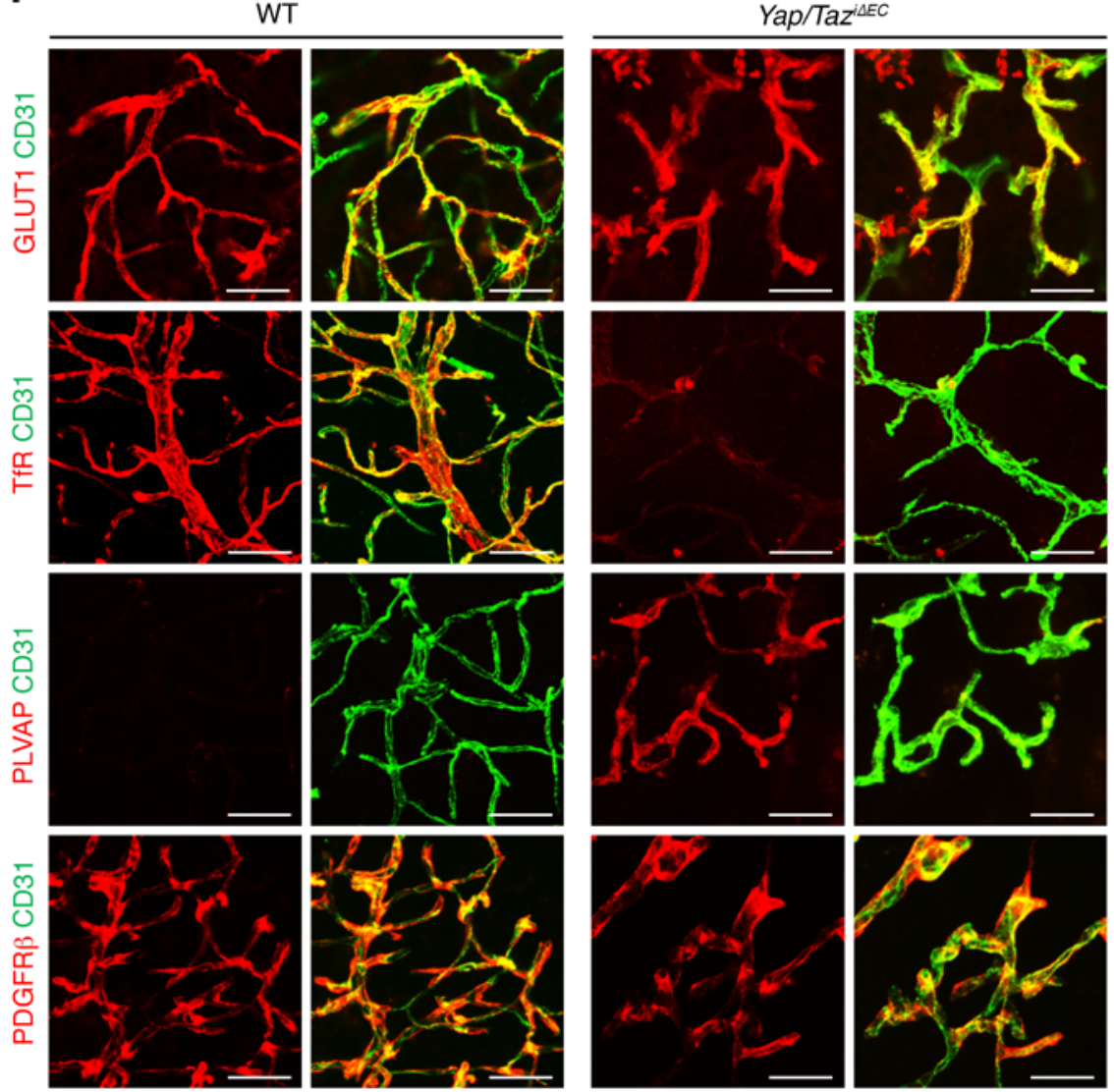

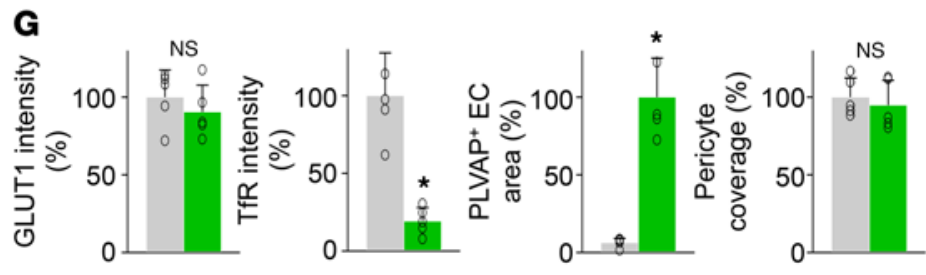

D
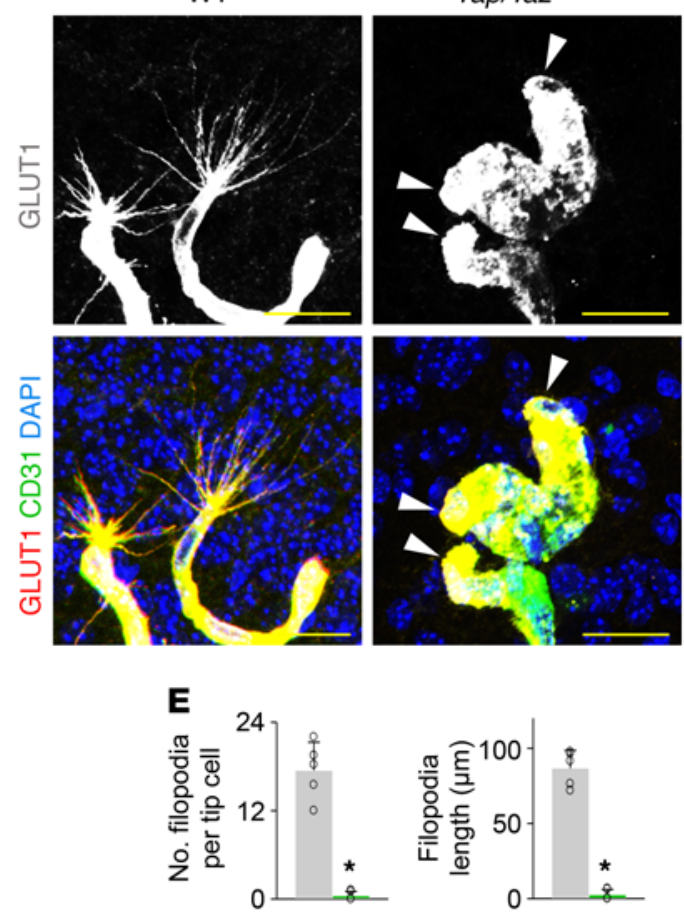

H

Yap/Taz ${ }^{\text {IAEC }}$ mouse

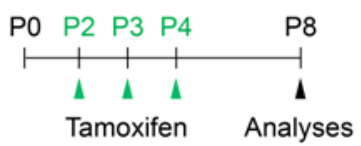

I

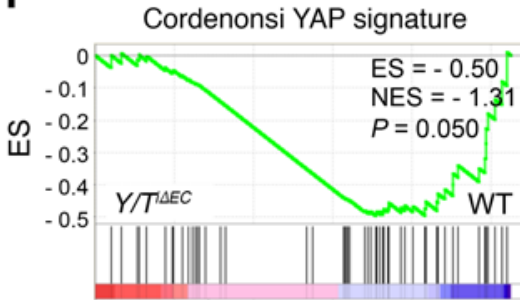

Enriched in WT then $Y / T^{\triangle E C}$

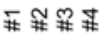

웅ㅇำ

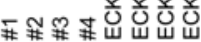

ミらららミミミミ

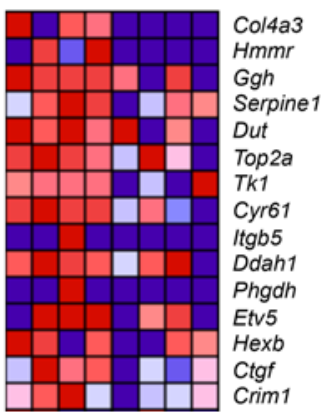


Figure 6. Endothelial YAP/TAZ is required for vascular network formation in brain. (A) Diagram for EC-specific deletion of Yap/Taz in brain vessels from $\mathrm{P} 2$ and their analyses at P12 in Yap/Taz ${ }^{\text {iAEC }}$ mice. (B and C) Images of $\mathrm{CD}^{+}{ }^{+}$vessels of cerebral cortex and striatum, and comparisons of indicated parameters in WT and Yap/Taz ${ }^{i \Delta E C}$ mice $(n=5$, each group). The yellow dashed line demarcates the border between cortex and striatum. Scale bars: $500 \mu \mathrm{m}$. (D and E) Magnified images of tip ECs and comparisons of indicated parameters in WT and Yap/Taz ${ }^{\text {iAEC }}$ mice ( $n=5$, each group). Tip ECs of Yap/Taz ${ }^{i \Delta E C}$ mice exhibit an aneurysm-like structure with less and dysmorphic filopodia (white arrowheads). Scale bars: $25 \mu \mathrm{m}$. (F and $\mathbf{G}$ ) Images and comparisons of levels of glucose transporter 1 (GLUT1), transferrin receptor (TfR), PLVAP, and PDGFR $\beta^{+}$pericyte coverage onto $C D 31^{+}$vessels in cerebral striatum of WT and Yap/Taz ${ }^{i \Delta E C}$ mice $(n=5$, each group). Scale bars: $50 \mu \mathrm{m}$. (H) Diagram for EC-specific deletion of Yap/Taz from P2 and sampling of brain ECs and their analyses at P8 in Yap/Taz ${ }^{\text {iAEC }}$ mice. (I) CSEA of isolated brain ECs showing downregulation of YAP signature genes in $\mathrm{Yap} / \mathrm{Taz}^{\mathrm{iSEC}}$ compared with those in WT mice, and correspondent heatmaps of the top 15 enriched genes. ES, enrichment score; NES, normalized enrichment score. Error bars represent mean $\pm \mathrm{SD}$. ${ }^{*} P<0.05$ vs. WT by Mann-Whitney $U$ test.

tized by frame grabber and reconstructed to images with a size of 512 $\times 512$ pixels per frame in real time. To visualize late-phase (6 minutes) FA and ICGA images using the angiography system, $10 \mathrm{mg}$ of fluorescein sodium (Alcon) and $0.15 \mathrm{mg}$ of indocyanine green (Daiichi Pharmaceutical) were administered i.p. and i.v., respectively. The imaging procedure was performed under systemic anesthesia and pupil dilation to improve the quality of images. Leaky areas from $\mathrm{CNV}$ were calculated as the total measured hyperfluorescent areas in FA images divided by the total measured CNV areas in ICGA images using Image ( $\mathrm{NIH}$ ).

Transmission electron microscopy. To visualize EC junction of brain vessels in high magnification, brain was harvested and sectioned after perfusion-fixation with $4 \%$ paraformaldehyde and $0.25 \%$ glutaraldehyde in $0.1 \mathrm{M}$ phosphate buffer ( $\mathrm{pH}$ 7.4). The tissues were then fixed overnight in $2.5 \%$ glutaraldehyde in $0.1 \mathrm{M}$ phosphate buffer ( $\mathrm{pH} 7.4$ ), postfixed with $1 \%$ osmium tetroxide, and dehydrated with series of increasing ethanol concentration followed by resin embedding. Ultrathin sections $(70 \mathrm{~nm})$ were obtained with an ultramicrotome (Leica, UltraCut UCT) and then collected on copper grids. After staining with $2 \%$ uranyl acetate and lead citrate, transmission electron microscopy was performed using a microscope (FEI Tecnai, G2 Spirit Twin) operated at $120 \mathrm{kV}$.

Electroretinography. Electroretinography was recorded to assess retinal neuronal function at the indicated time points. Mice were either dark- or light-adapted for 12 hours before electroretinographic monitoring, anesthetized, and placed on a heating pad to maintain body temperature. After dilation of pupils with $1 \%$ tropicamide and $2.5 \%$ phenylephrine eye drops (Santen Pharmaceutical), cornea was placed on a gold-plated objective lens, while silver-embedded needle electrodes were placed at forehead (reference) and tail (ground). The electroretinographic stimulus and recording were performed using LabScribe ERG version 3 software combined with a retinal imaging microscope (Phoenix Research Labs, Micron IV) following the manufacturer's instructions. To obtain scotopic (dark-adapted condition) a- and bwaves, a digital bandpass filter ranging from 0.3 to $1,000 \mathrm{~Hz}$ and stimulus ranging from -2.2 to $2.2 \log \left(\mathrm{cd} \cdot \mathrm{s} / \mathrm{m}^{2}\right)$ were used. To obtain photopic (light-adapted condition) b-waves, a filter ranging from 2 to $200 \mathrm{~Hz}$ and stimulus ranging from 0.4 to $2.2 \log \left(\mathrm{cd} \cdot \mathrm{s} / \mathrm{m}^{2}\right)$ with $1.3 \log \left(\mathrm{cd} \cdot \mathrm{s} / \mathrm{m}^{2}\right)$ background were used. After averaging of the signals, the amplitudes of a- and b- waves were presented by the LabScribe ERG version 3 software and used for analyses.

Cell culture. Pooled primary cultured HUVECs were purchased from Lonza. HUVECs were cultured in EGM-2 media (Lonza) in culture dishes coated with $0.1 \%$ gelatin. HUVECs at passages 3-6 were used for this study.

RNA interference. HUVECs were transfected with a pool of siRNAs using Lipofectamine RNAiMAX (Invitrogen) according to the manufacturer's protocols. The following target sequences were used: human YAP (5'-GACAUCUUCUGGUCAGAGA-3') and human TAZ (5'-ACGUUGACUUAGGAACUUU-3'). GL2 siRNA (5'-CGTACGCGGAATACTTCGA-3') was used as negative control. Cells were harvested 48-72 hours after transfection. Efficiency of knockdown was evaluated by immunoblotting.

Cell growth assay and cell cycle analysis. Cell proliferation was measured using the Cell Counting Kit-8 reagent (CCK-8, Dojindo). HUVECs were seeded into a 96 -well plate at $3 \times 10^{3}$ cells per well and cultured for 24 hours. Then, $10 \mu \mathrm{lCCK}-8$ was treated to each well after 0, 24, 48, and 72 hours. Measurements were taken at the absorbance of $450 \mathrm{~nm}$ at 2 hours after treatment of CCK-8. For cell cycle analysis, HUVECs were incubated with $10 \mu \mathrm{M}$ BrdU for 4 hours and were processed with the APC BrdU flow kit (BD Biosciences) following the manufacturer's protocols. The cell cycle profiles were characterized with a FACS Canto II (BD Biosciences) and analyzed with FlowJo (Tree Star).

Metabolic assays. The extracellular acidification rate (ECAR) and oxygen consumption rate (OCR) were measured using the Seahorse XFe96 analyzer (Seahorse Bioscience) following the manufacturer's instructions. Briefly, ECAR and OCR were measured 6 hours after seeding of HUVECs (20,000 cells per well) on $0.1 \%$ gelatin-coated XFe96 microplates. HUVECs were maintained in nonbuffered assay medium in a non- $\mathrm{CO}_{2}$ incubator for 1 hour before the assay. The Glycolysis stress test kit (Seahorse Bioscience) was used to monitor ECAR under various conditions. Three baseline recordings were made, followed by sequential injections of glucose $(10 \mathrm{mM})$, the mitochondrial/ATP synthase inhibitor oligomycin $(3 \mu \mathrm{M})$, and the glycolysis inhibitor 2-deoxy-Dglucose (100 mM). The Mito stress test kit (Seahorse Bioscience) was used to assay the OCR under basal conditions in the presence of oligomycin $(3 \mu \mathrm{M})$, the mitochondrial uncoupler carbonyl cyanide-4(trifluoromethoxy)phenylhydrazone (FCCP; $1 \mu \mathrm{M}$ ), and the respiratory chain inhibitors rotenone and antimycin $\mathrm{A}(1.5 \mu \mathrm{M})$.

Measurement of the GTP-bound forms of RhoA, Rac1, and CDC42. HUVECs were incubated for 24 hours in EBM-2 media (Lonza) to starve the cells, and then treated with $50 \mathrm{ng} / \mathrm{ml}$ VEGF for $30 \mathrm{~min}$ utes. Cell lysates were collected, and levels of activated GTP-bound RhoA, Rac1, and CDC42 were determined using the G-LISA RhoA (BK121), Rac1 (BK126), and CDC42 (BK127) Activation Assay Biochem Kits (Cytoskeleton Inc.).

Wound healing assay. The migration of HUVECs was measured using a Culture-Insert 2 Well (ibidi $\mathrm{GmbH}$ ) according to the manufacturer's instructions. Briefly, 48-72 hours after siRNA transfection, cells were seeded into culture inserts and were incubated to allow them to adhere. The culture insert provided 2 cell culture reservoirs that were separated by an approximately $500-\mu \mathrm{m}$-thick wall. After removal of the culture inserts on the second day, a "wound" of approximately $500 \mu \mathrm{m}$ was formed between the 2 cell patches. Photos 
A

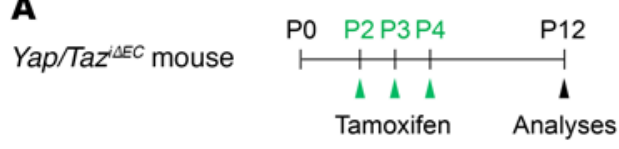

B
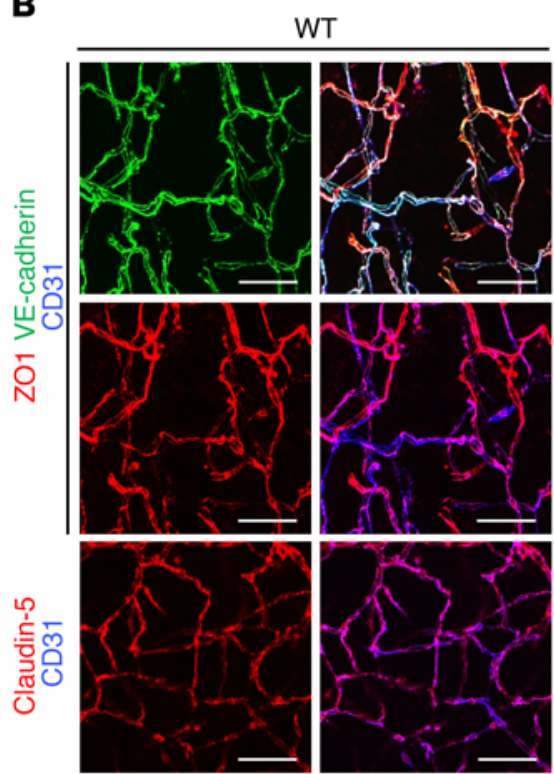

E
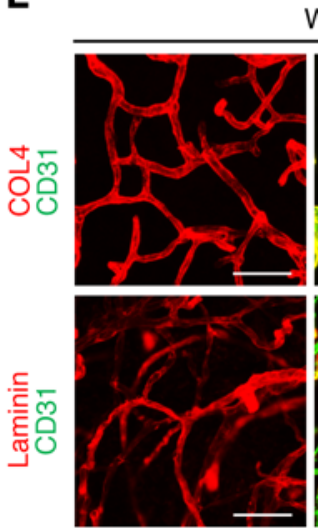

WT
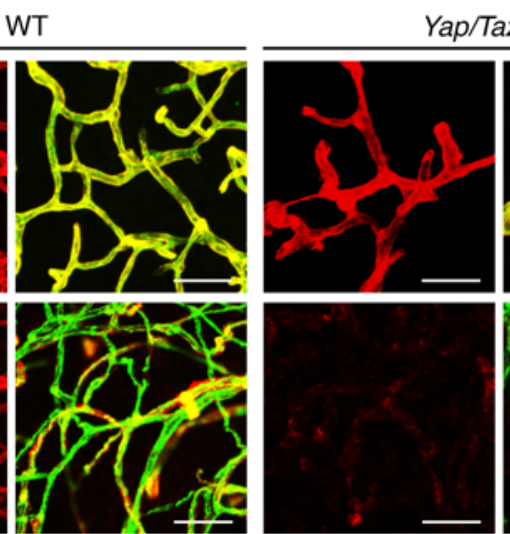

Yap/Taz ${ }^{i \Delta E C}$
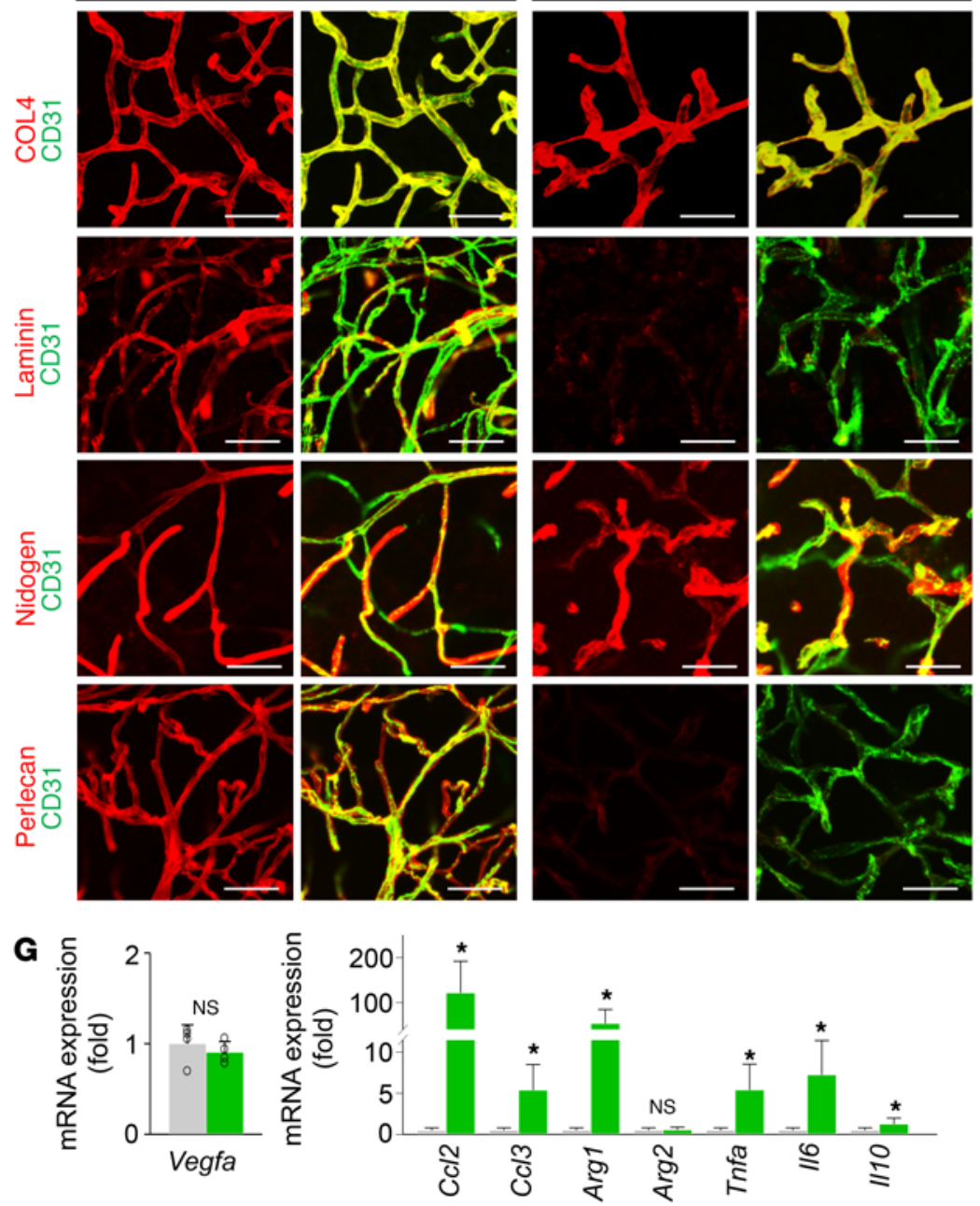

C

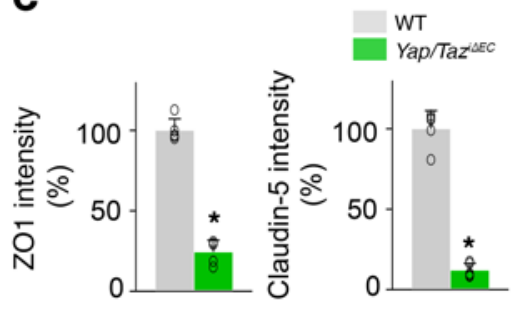

D

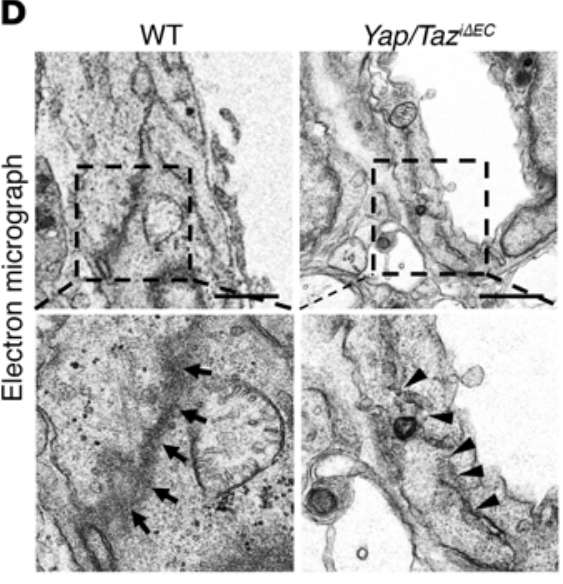

F
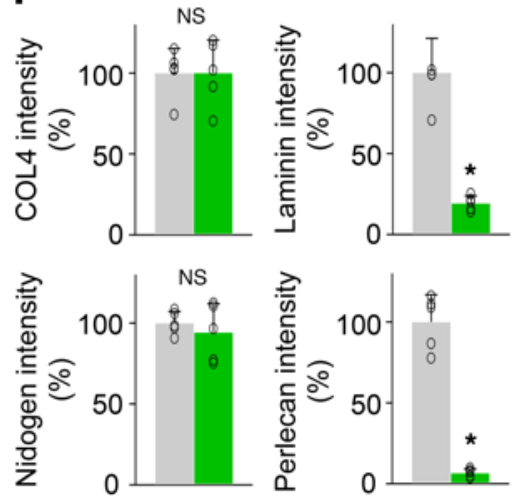

H GO regulation of cell junction assembly

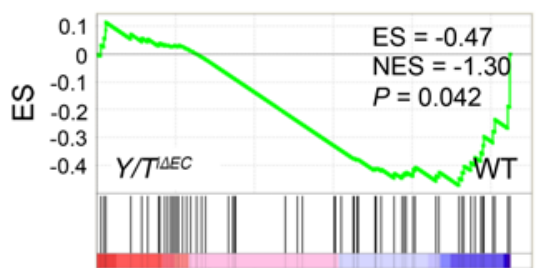

Enriched in WT then $Y / T^{\triangle E C}$

GO extracellular structure organization

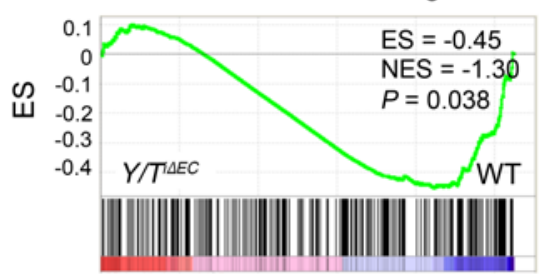

Enriched in WT then $Y / T^{\triangle E C}$ 
Figure 7. Endothelial YAP/TAZ is indispensable for BBB maturation. (A) Diagram for EC-specific deletion of Yap/Taz in brain vessels from P2 and their analyses at P12 in Yap/Taz ${ }^{i E C}$ mice. (B and C) Images and comparisons of distributions of VE-cadherin, Z01, and claudin-5 on CD31 ${ }^{+}$vessels in cerebral striatum of WT and Yap/TaziLEC mice ( $n=5$, each group). Scale bars: $50 \mu \mathrm{m}$. (D) Electron microscopic images showing electron-dense and continuous junction between brain ECs in WT mice (arrows), while junction between ECs is disrupted and less electron-dense in Yap/Tazi ${ }^{i L C}$ mice (arrowheads). Scale bars: $1 \mu \mathrm{m}$. (E and F) Images and comparisons of levels of COL4, laminin, nidogen, and perlecan on CD31+ vessels in cerebral striatum of WT and Yap/Taz ${ }^{i \Delta E C}$ mice $(n=5$, each group). Scale bars: $50 \mu \mathrm{m}$. (G) Comparisons of expressions of indicated mRNA in brains of WT and Yap/Taz ${ }^{i \Delta E C}$ mice $(n=4$, each group). (H) GSEA showing

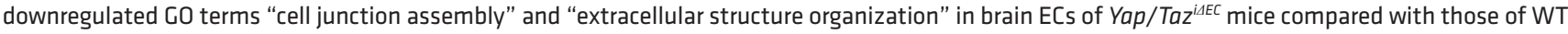
mice. ES, enrichment score; NES, normalized enrichment score. Error bars represent mean \pm SD. ${ }^{*} P<0.05$ vs. WT by Mann-Whitney $U$ test.

of the wound were taken after 12 hours. The widths of the wounds were analyzed using ImageJ.

Isolation of brain and lung ECs. After anesthesia, brain was harvested at P8, cut into small pieces, and digested in buffer containing $0.1 \%$ collagenase type $1 \mathrm{~A}$ (Worthington) and $3 \mathrm{U} / \mathrm{ml}$ DNase I (Roche) at $37^{\circ} \mathrm{C}$ for 45 minutes. Tissues were gently agitated, strained with a $40-\mu \mathrm{m}$ nylon mesh to remove cell clumps, and incubated in ACK lysis buffer for 2 minutes to remove erythrocytes. Single-cell suspensions were incubated for 30 minutes with biotin-conjugated rat antiCD31 (Miltenyi Biotec, 130-101-955), washed to remove unbound antibody, and incubated for 20 minutes with anti-biotin microbeads (Miltenyi Biotec, 130-090-485). CD31 ${ }^{+}$cells were enriched using an AutoMACS Pro Separator (Miltenyi Biotec). Enriched cells were further stained with the following antibodies: PE-conjugated rat antiCD31 (BD Pharmingen, 553373), PE-conjugated streptavidin (eBioscience, 12-4317), APC-conjugated rat anti-CD45 (BD Pharmingen,
559864), and APC-conjugated rat anti-TER119 (BD Pharmingen, 557909). To discriminate dead cells, cells were stained with DAPI (Sigma-Aldrich) and cell sorting was performed with FACSAriaII (BD Biosciences). Data were analyzed using FlowJo software (Tree Star). Lung EC isolation was performed with the same method as described above but without enrichment of CD $31^{+}$cells by AutoMACS.

RNA sequencing and accession numbers. After extraction of total RNA from cells using Trizol reagent (Invitrogen), RNA quality was assessed by an Agilent 2100 bioanalyzer using the RNA 6000 Nano Chip (Agilent Technologies), and RNA quantification was performed using an ND-2000 Spectrophotometer (Thermo Fisher Scientific). RNA sequencing (RNA-Seq) was performed with TruSeq RNA Library Prep kit v2 and NextSeq 500 equipment (Illumina). Mapping of RNA-Seq reads was performed using the TopHat software tool so as to acquire the alignment file, which was used to bring together transcripts, assess their exuberances, and identify
A
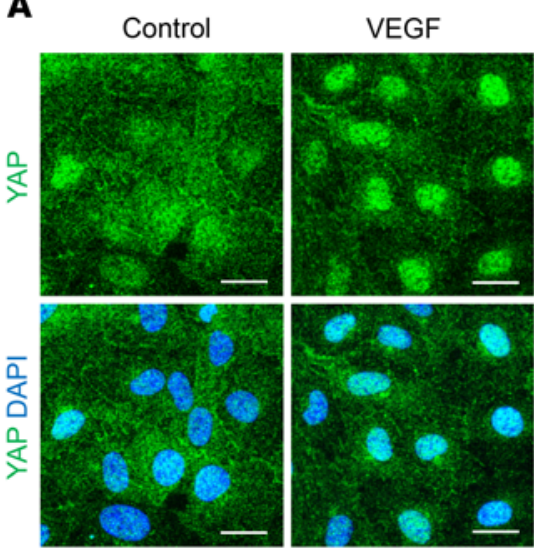

B

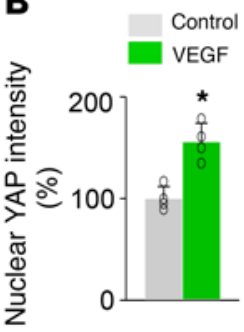

C

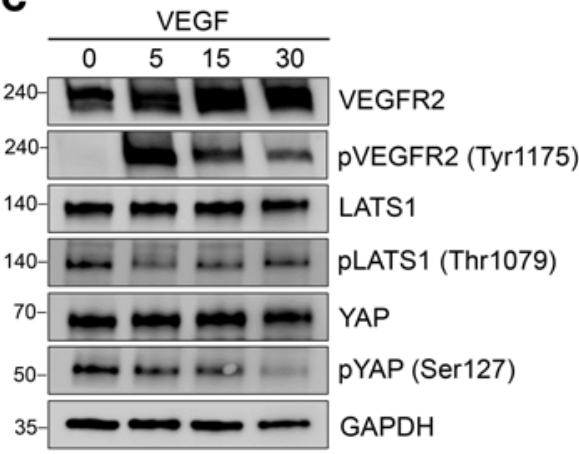

$\mathbf{F}$

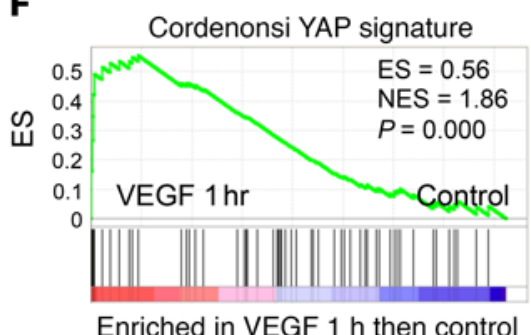

Figure 8. VEGF upregulates YAP/TAZ transcriptional activity in ECs. (A and B) Images and quantification of the nuclear enrichment of YAP in HUVECs stimulated with VEGF ( $50 \mathrm{ng} / \mathrm{ml}$ ) for 30 minutes ( $n=4$, each group). Scale bars: $20 \mu \mathrm{m}$. (C and D) Immunoblot analyses and comparisons of indicated proteins in HUVECs stimulated with VEGF $(50 \mathrm{ng} / \mathrm{ml}$ ) for indicated times ( $n=3$, each group). Note that, upon VEGFR2 activation, the level of pLATS1 (Thr1079) is reduced after 5 minutes, and reduction in PYAP (Ser127) level follows after 30 minutes. (E) Quantitative PCR analysis of CTCF and CYR61 mRNA levels in HUVECs stimulated with VEGF for 1 hour $(n=4$, each group). (F) GSEA of the microarray data (GSE18913) showing upregulation of YAP signature genes in HUVECs stimulated with VEGF for 1 hour compared with control. ES, enrichment score; NES, normalized enrichment score. Error bars and dots represent mean \pm SD. ${ }^{*} P<0.05$ vs. control or 0 minutes by Mann-Whitney $U$ test. 

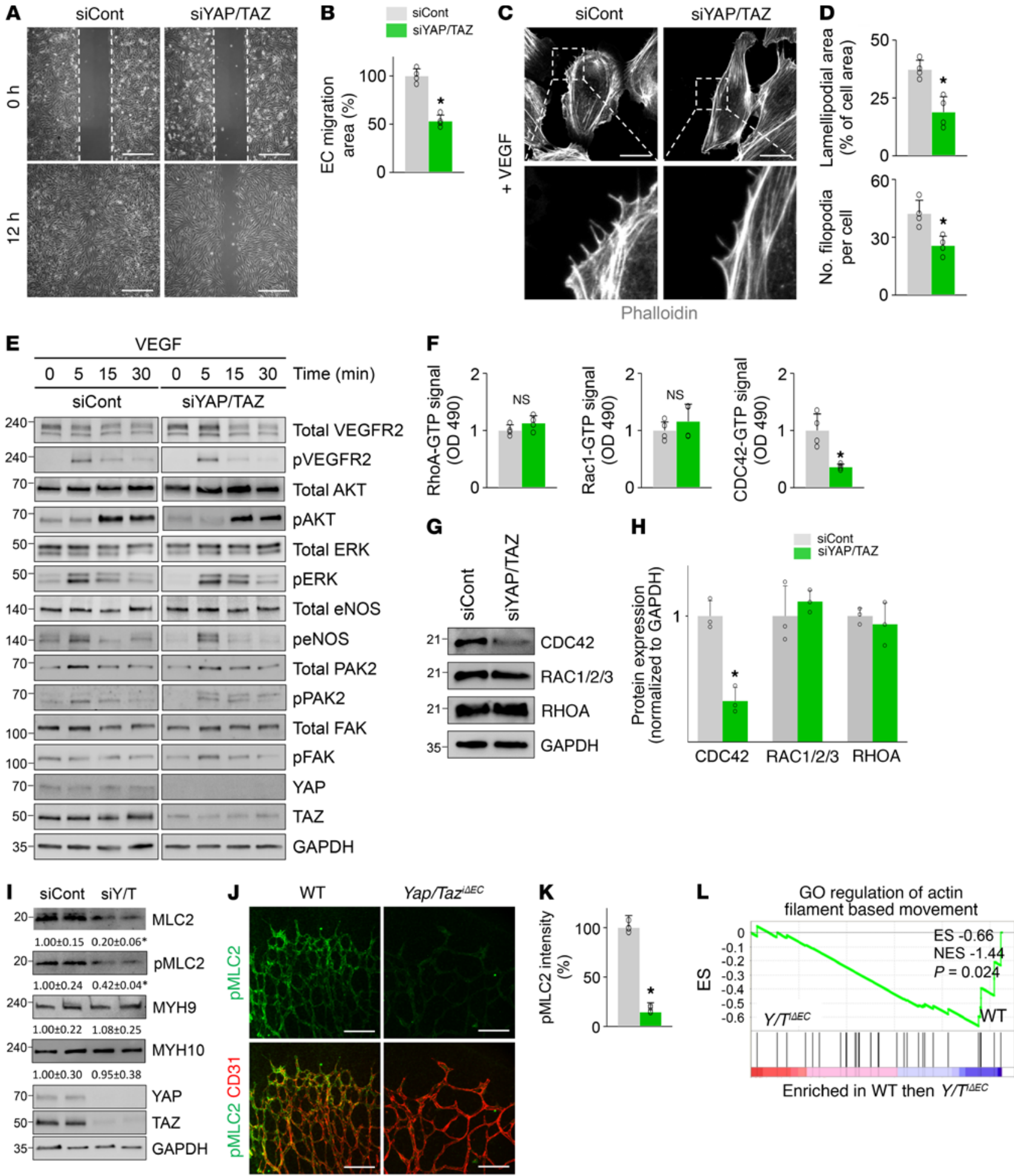

Enriched in WT then $Y / T T^{\triangle E C}$

Figure 9. YAP/TAZ depletion impairs EC migration and filopodia/lamellipodia formation by suppressing CDC42 and MLC2 activity. (A and B) Images and comparison of wound healing migration in HUVECs transfected with siCont (siCont-ECs) or siYAP/TAZ (siYAP/TAZ-ECs) ( $n=4$, each group). Scale bars: 500 $\mu \mathrm{m}$. (C and $\mathbf{D})$ Images of phalloidin ${ }^{+}$actin cytoskeleton and comparisons of indicated parameters in siCont-ECs and siYAP/TAZ-ECs after VEGF stimulation for 30 minutes ( $n=4$, each group). Scale bars: $20 \mu \mathrm{m}$. (E) Immunoblot analyses of indicated proteins in siCont-ECs and siYAP/TAZ-ECs stimulated with VECF for indicated times. Similar findings were observed in 3 independent experiments. (F) Comparisons of activities of RhoA, Rac1, and CDC42 in siCont-ECs and siYAP/TAZ-ECs stimulated with VEGF for 30 minutes ( $n=4$, each group). Note that siYAP/TAZ-ECs show markedly attenuated CDC42 activity. (G and $\mathbf{H}$ ) Immunoblot analyses and comparisons of indicated proteins in siCont-ECs and siYAP/TAZ-ECs ( $n=3$, each group). Note that siYAP/TAZ-ECs show selectively reduced protein level of CDC42. (I) Immunoblot analyses and comparisons of indicated proteins in siCont-ECs and siYAP/TAZ-ECs (siY/T) ( $n=3-4$, each group). Numbers indicate mean \pm SD. Note that siYAP/TAZ-ECs show significantly reduced MLC2, a key regulator of contractile force. (J and $\mathbf{K})$ Images and comparison of pMLC2 (at Ser19) in CD31+ retinal vessels at P5 in WT and Yap/Taz ${ }^{\text {iAEC }}$ mice ( $n=4$, each group). Scale bars: $100 \mu \mathrm{m}$. (L) GSEA of isolated brain ECs showing downregulated GO term "regulation of actin filament based movement" in Yap/Taz ${ }^{i \in C}$ mice compared with WT mice. ES, enrichment score; NES, normalized enrichment score. Error bars represent mean \pm SD. ${ }^{*} P<0.05$ vs. siCont or WT mice by Mann-Whitney $U$ test. 

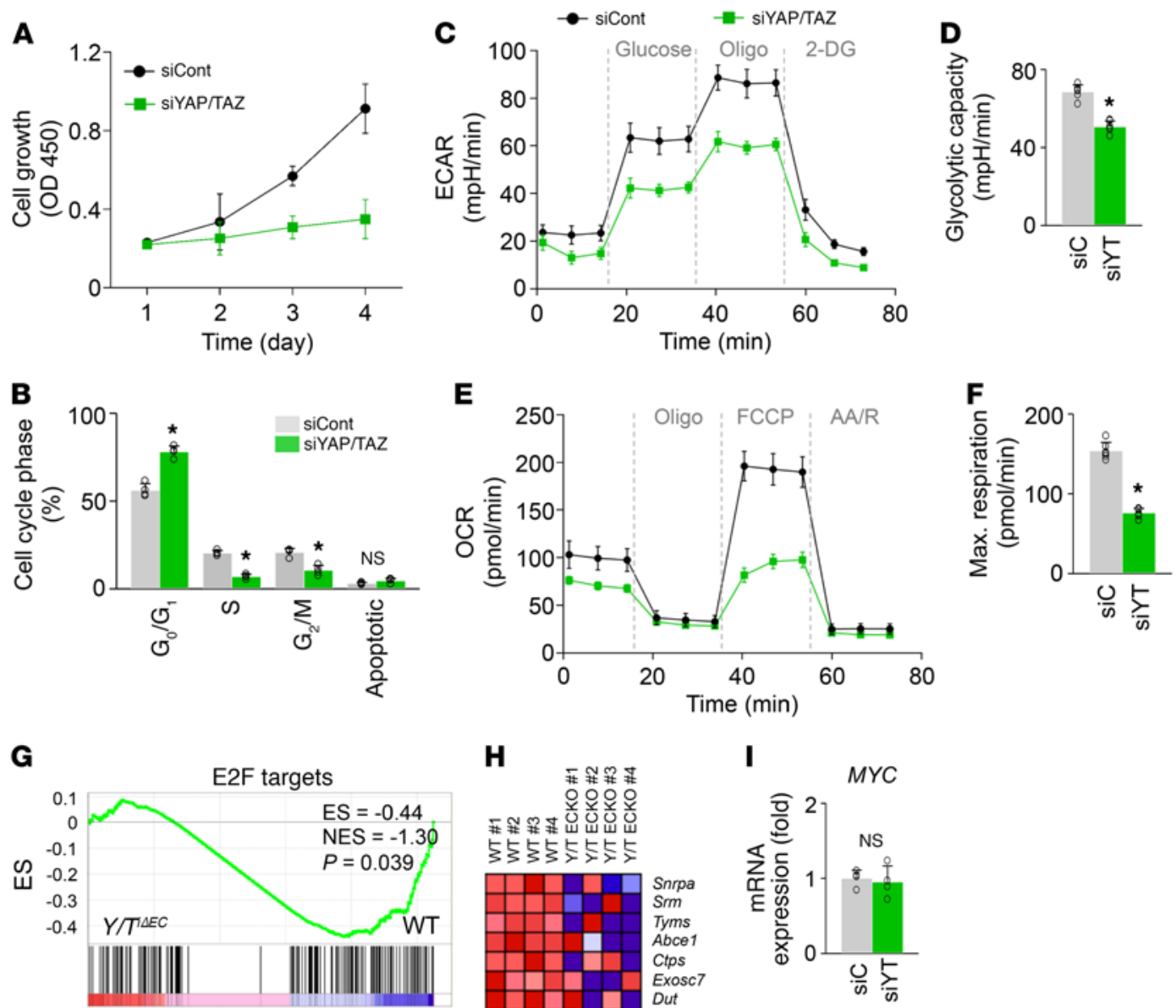

H

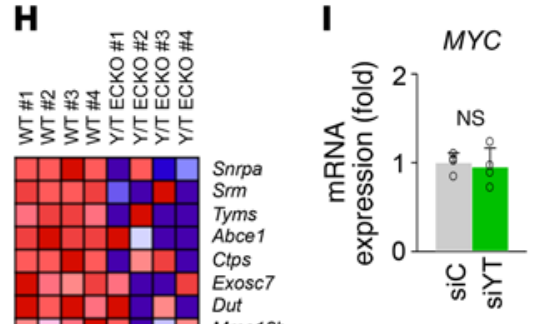

Enriched in WT then $Y / T^{\triangle E E C}$

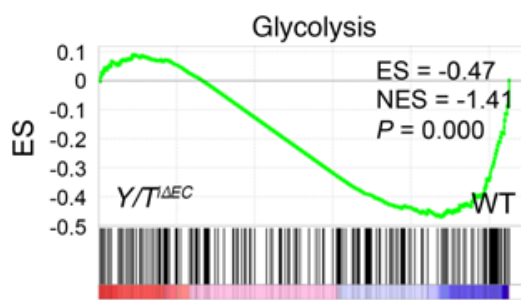

Enriched in WT then $Y / T^{\triangle E C}$

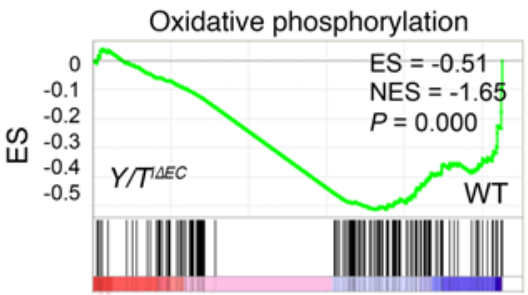

Enriched in WT then $Y / T \Delta E C$
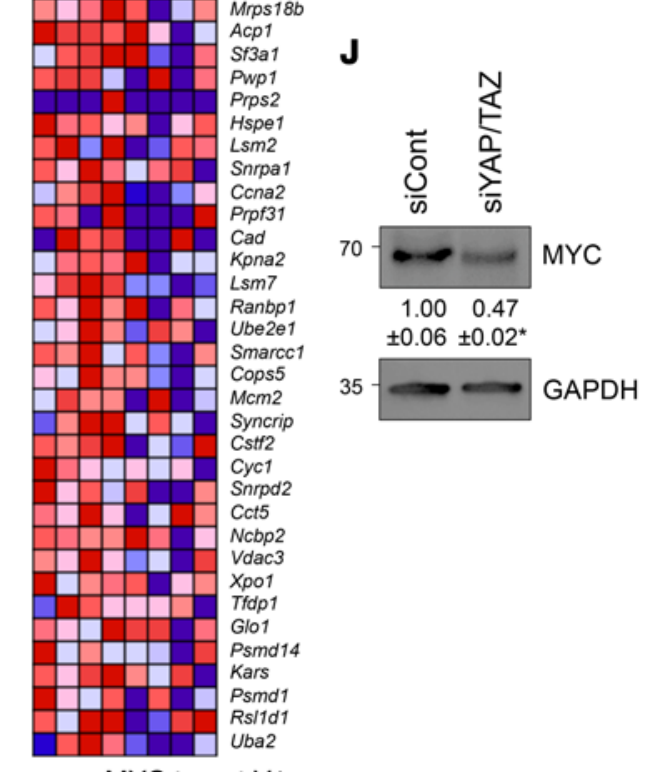

Figure 10. YAP/TAZ positively regulates proliferation and metabolic activity of ECs through MYC signaling. (A and B) Growth and cell cycle analysis of HUVECs transfected with siCont (siCont-ECs) or siYAP/TAZ (siYAP/TAZ-ECs) ( $n=4$, each group). (C and D) Comparisons of extracellular acidification rate (ECAR) of siCont-ECs (siC) and siYAP/TAZ-ECs (siYT) under basal conditions and in response to glucose, oligomycin (Oligo), and 2-deoxy-D-glucose (2-DC) ( $n=5$, each group). (E and $\mathbf{F}$ ) Comparisons of oxygen consumption rate (OCR) of siCont-ECs and siYAP/TAZ-ECs under basal conditions and in response to oligo, fluoro-carbonyl cyanide phenylhydrazone (FCCP), and antimycin A (AA)/rotenone (R) ( $n=5$, each group). (G) GSEA of isolated brain ECs showing downregulations of E2F target, glycolysis, and oxidative phosphorylation signature genes in Yap/Taz ${ }^{i \Delta E C}$ compared with WT mice. ES, enrichment score; NES, normalized enrichment score. (H) Heatmap of MYC signature genes of brain ECs sorted from WT and Yap/TaziLEC mice. (I and J) Quantitative PCR ( $n=4$, each group) and immunoblot analysis of MYC expression in siCont-ECs and siYAP/TAZ-ECs. Error bars represent mean \pm SD. ${ }^{*} P<0.05$ vs. siCont by Mann-Whitney $U$ test. 
A

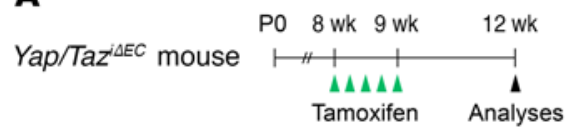

B

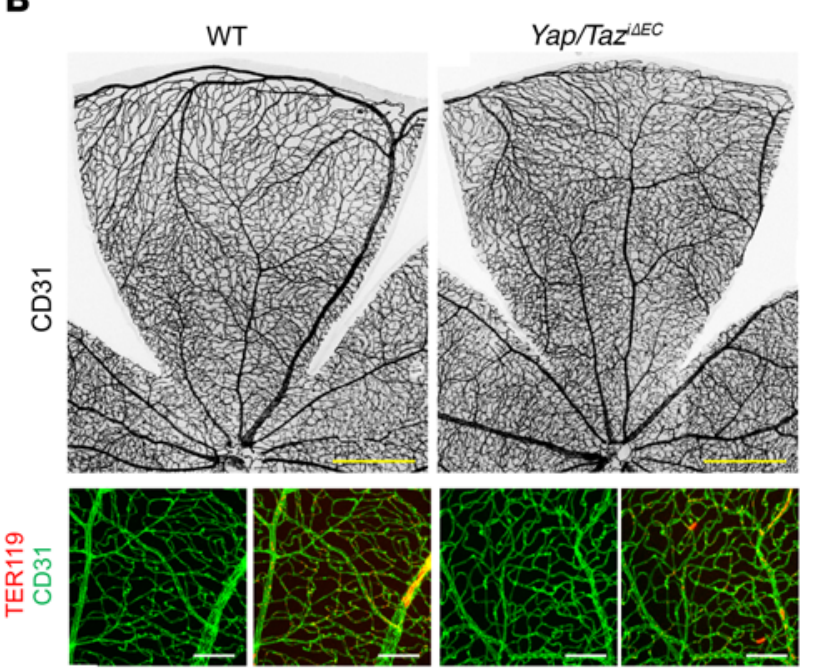

E
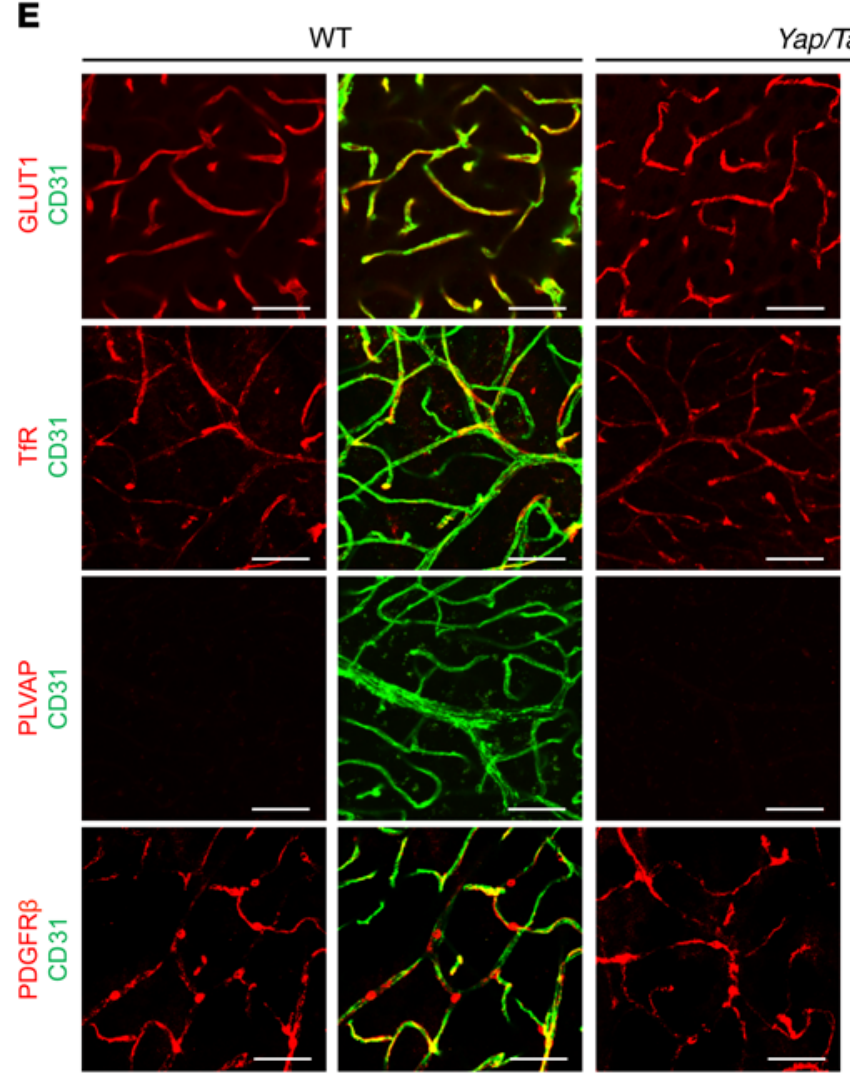

Yap/Taz ${ }^{\mathrm{iEC}}$

$\mathbf{F}$

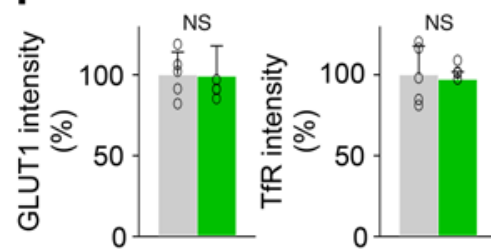

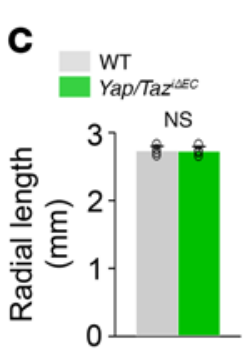

D

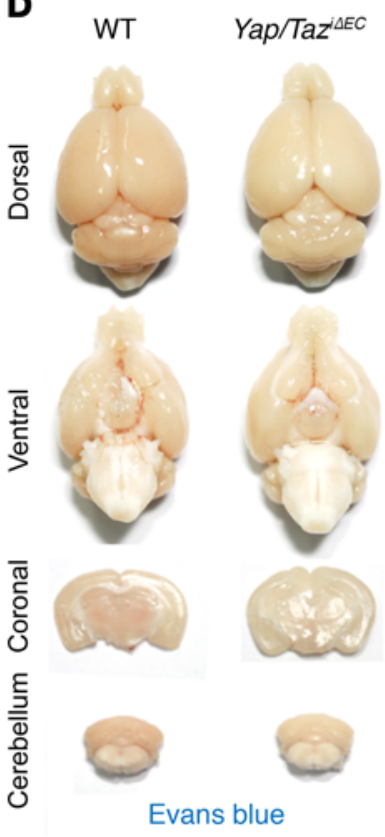

G

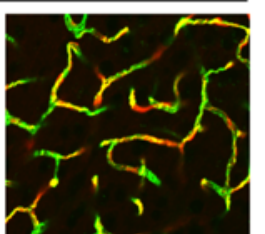

Yap/Taz ${ }^{-\triangle E C}$ mouse

H
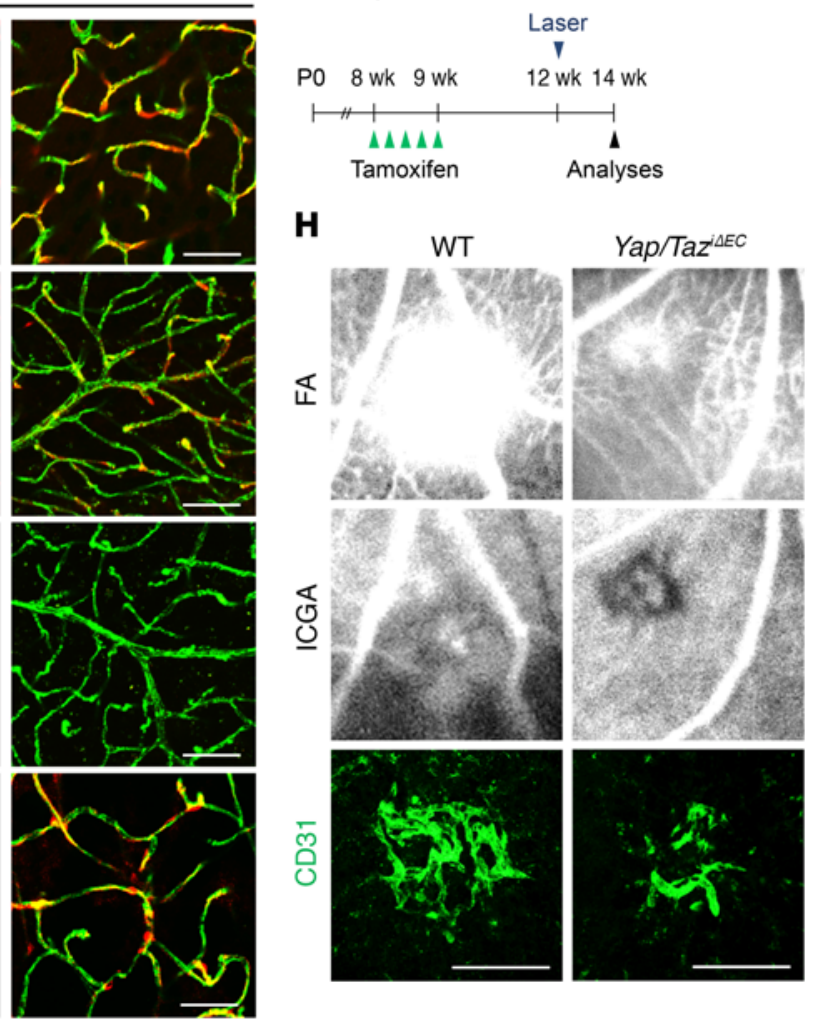
Figure 11. Endothelial YAP/TAZ is required for pathological angiogenesis, but dispensable for the maintenance of barrier integrity during adulthood. (A) Diagram for EC-specific deletion of Yap/Taz in retinal and brain vessels of 8-week-old mice and their analyses after 4 weeks in Yap/Taz ${ }^{i L E C}$ mice. (B and C) Images of $\mathrm{CD} 1^{+}$retinal vessels and comparisons of indicated parameters in WT and Yap/Taz ${ }^{\text {iEEC }}$ mice ( $n=4$, each group). Scale bars: $500 \mu \mathrm{m}$, top panels; $100 \mu \mathrm{m}$, bottom panels. (D) Images of whole and sectioned brains from WT and Yap/TazitEC mice. No visible hemorrhage or EB leakage is detected in both mice. (E and F) Images and comparisons of levels of CLUT1, TfR, PLVAP, and PDGFR $\beta^{+}$pericyte coverage onto CD31+ vessels in cerebral striatum of WT and Yap/Taz ${ }^{\text {isEC }}$ mice $(n=5$, each group). Scale bars: $50 \mu \mathrm{m}$. (C) Diagram for EC-specific deletion of Yap/Taz in retinal vessels of 8-week-old mice, generation of choroidal neovascularization (CNV) 4 weeks later, and their analyses at 2 weeks after laser photocoagulation in Yap/Taz ${ }^{i \Lambda E C}$ mice. (H) Images of late-phase fluorescein angiography (FA) for detecting vascular leakage surrounding the site of laser injury, and indocyanine green angiography (ICGA) and CD31 staining of retinal pigment epithelium-choroid-sclera flat mounts for quantifying the extent of CNV in WT and Yap/Taz ${ }^{\text {iEEC }}$ mice. Scale bars: $100 \mu \mathrm{m}$. (I) Comparisons of CNV volumes calculated by total measurements of CD31+ CNV volume, and leaky areas from CNV calculated as total measured hyperfluorescent areas in FA images divided by total measured CNV areas in ICGA images, in WT and Yap/Taz ${ }^{i L C}$ mice $\left(n=5\right.$, each group). Error bars represent mean \pm SD. ${ }^{*} P<0.05$ vs. WT by Mann-Whitney $U$ test.

differentially expressed genes or isoforms using cufflinks. All gene expression values from RNA-Seq were changed to $\log _{2}$ values and analyzed further. Then gene set enrichment analysis (GSEA) was performed with version 5.2 of the Molecular Signatures Database (http://www.broadinstitute.org/gsea/msigdb), and the gene sets that had less than 0.05 nominal $P$ value were stated. The original data are available in the National Center for Biotechnology Information's Gene Expression Omnibus (accession nos. GSE94673, brain ECs, and GSE94685, lung ECs).

Statistics. Values are presented as mean \pm SD. Statistical significance was determined by the 2-sided Mann-Whitney $U$ test between 2 groups or the Kruskal-Wallis test followed by Tukey's honest significant difference test with ranks for multiple-group comparison. Survival curve was evaluated using the Kaplan-Meier method, and statistical differences were analyzed using the log-rank test. Statistical analysis was performed using GraphPad Prism 7.0 (GraphPad Software). Statistical significance was set to $P$ value less than 0.05 .

Study approval. Animal care and experimental procedures were performed under approval from the Institutional Animal Care and Use Committee (no. KA2016-39) of Korea Advanced Institute of Science and Technology, Daejeon, South Korea. Mice were handled in accordance with the Association for Research in Vision and Ophthalmology Statement for the Use of Animals in Ophthalmic and Vision Research.

\section{Author contributions}

JoK and GYK designed the research studies. JoK, YHK, JaK, DYP, $\mathrm{HB}, \mathrm{DHL}, \mathrm{KHK}, \mathrm{SPH}$, and SPJ conducted the experiments and analyzed the data. YK provided the mice, and JoK, YHK, and GYK generated the figures and wrote and edited the manuscript. YGK, DSL, and GYK directed and supervised the project.

\section{Acknowledgments}

We thank Sun-Hye Jeong and Wonyoung Choi for reagents and comments. We thank Intae Park for proofreading of the manuscript. We also thank Sujin Seo and Hyun Tae Kim for their technical assistance. This study was supported by the Institute for Basic Science funded by the Ministry of Science, ICT and Future Planning, Korea (IBS-R025-D1-2015 to GYK).

Address correspondence to: Gou Young Koh, Center for Vascular Research, IBS, Graduate School of Medical Science and Engineering, KAIST, 291 Daehak-ro, Daejeon 34141, South Korea. Phone: 82.42.350.2638; Email: gykoh@kaist.ac.kr. Or to: Dae-Sik Lim, National Creative Research Initiatives Center for Cell Division and Differentiation, Department of Biological Science, KAIST, 291 Daehak-ro, Daejeon 34141, South Korea. Phone: 82.42.350.2635; Email: daesiklim@kaist.ac.kr.
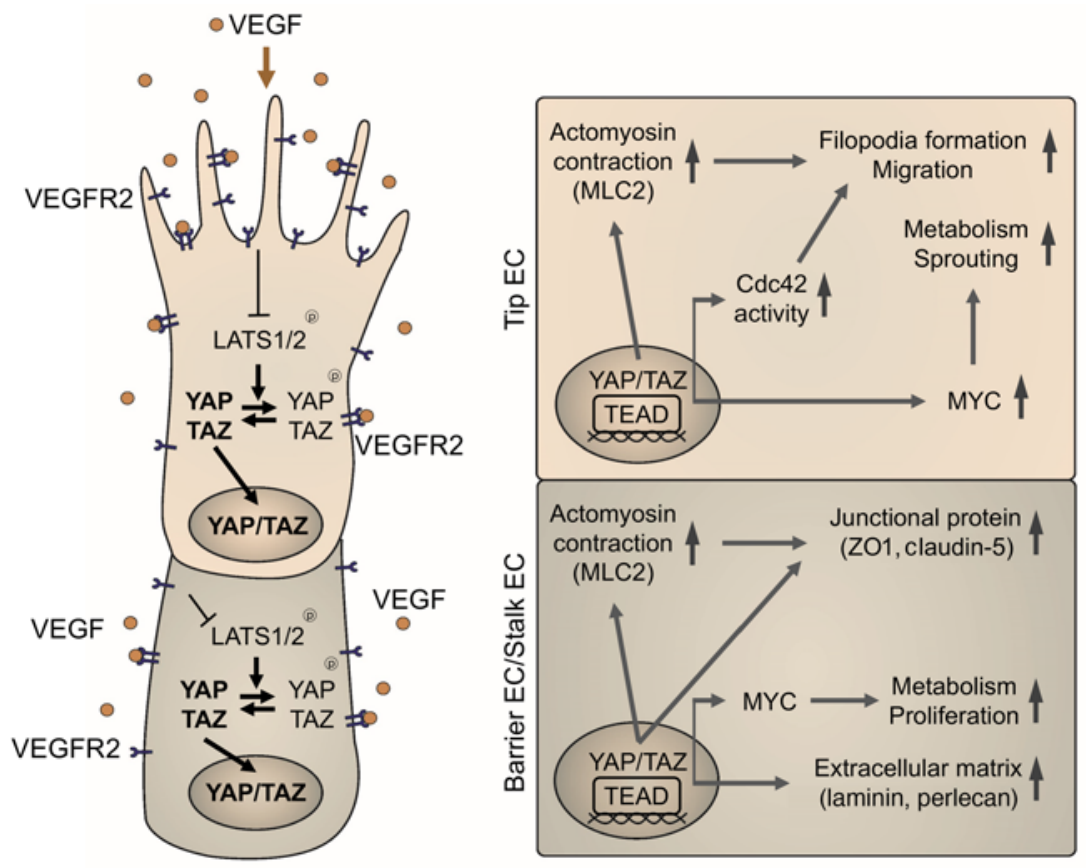

Figure 12. Schematic diagram depicting the roles of YAP/TAZ in sprouting angiogenesis and vascular barrier maturation. In response to VEGF, YAP/ TAZ binds to TEAD family transcriptional factors, and positively controls activity of Cdc42 and MLC2 for actomyosin contractility, which are essential for filopodia formation and cell migration in tip ECs. Simultaneously, YAP/TAZ upholds expression and activity of MYC signaling, which is a key driver of EC metabolism and growth, thereby promoting cell proliferation in stalk ECs and maturation in barrier ECs forming the BBB. Along with angiogenic sprouting, YAP/TAZ also promotes formation and maturation of vascular barrier by upregulating expressions of junctional molecules and ECM components. 
1. Potente M, Gerhardt H, Carmeliet P. Basic and therapeutic aspects of angiogenesis. Cell. 2011;146(6):873-887.

2. Chung AS, Ferrara N. Developmental and pathological angiogenesis. Annu Rev Cell Dev Biol. 2011;27:563-584.

3. Blanco R, Gerhardt H. VEGF and Notch in tip and stalk cell selection. Cold Spring Harb Perspect Med. 2013;3(1):a006569.

4. Geudens I, Gerhardt H. Coordinating cell behaviour during blood vessel formation. Development. 2011;138(21):4569-4583.

5. Eilken HM, Adams RH. Dynamics of endothelial cell behavior in sprouting angiogenesis. Curr Opin Cell Biol. 2010;22(5):617-625.

6. Yu FX, Zhao B, Guan KL. Hippo pathway in organ size control, tissue homeostasis, and cancer. Cell. 2015;163(4):811-828.

7. Piccolo S, Dupont S, Cordenonsi M. The biology of YAP/TAZ: hippo signaling and beyond. Physiol Rev. 2014;94(4):1287-1312.

8. Dupont S, et al. Role of YAP/TAZ in mechanotransduction. Nature. 2011;474(7350):179-183.

9. Gumbiner BM, Kim NG. The Hippo-YAP signaling pathway and contact inhibition of growth. J Cell Sci. 2014;127(pt 4):709-717.

10. Halder G, Dupont S, Piccolo S. Transduction of mechanical and cytoskeletal cues by YAP and TAZ. Nat Rev Mol Cell Biol. 2012;13(9):591-600.

11. Mahoney JE, Mori M, Szymaniak AD, Varelas $\mathrm{X}$, Cardoso WV. The hippo pathway effector Yap controls patterning and differentiation of airway epithelial progenitors. Dev Cell. 2014;30(2):137-150.

12. Reginensi A, Hoshi M, Boualia SK, Bouchard M, Jain S, McNeill H. Yap and Taz are required for Ret-dependent urinary tract morphogenesis. Development. 2015;142(15):2696-2703.

13. Dai X, et al. Phosphorylation of angiomotin by Lats1/2 kinases inhibits F-actin binding, cell migration, and angiogenesis. J Biol Chem. 2013;288(47):34041-34051.

14. Morin-Kensicki EM, et al. Defects in yolk sac vasculogenesis, chorioallantoic fusion, and embryonic axis elongation in mice with targeted disruption of Yap65. Mol Cell Biol. 2006;26(1):77-87.

15. Lee JH, et al. A crucial role of WW45 in developing epithelial tissues in the mouse. EMBO J. 2008;27(8):1231-1242.

16. Oh S, et al. Crucial role for Mst1 and Mst2 kinases in early embryonic development of the mouse. Mol Cell Biol. 2009;29(23):6309-6320.

17. Choi HJ, et al. Yes-associated protein regulates endothelial cell contact-mediated expression of angiopoietin-2. Nat Commun. 2015;6:6943.

18. Giampietro C, et al. The actin-binding protein EPS8 binds VE-cadherin and modulates YAP localization and signaling. J Cell Biol. 2015;211(6):1177-1192.

19. Wang KC, et al. Flow-dependent YAP/TAZ activities regulate endothelial phenotypes and atherosclerosis. Proc Natl Acad Sci U S A. 2016;113(41):11525-11530.

20. Wang L, et al. Integrin-YAP/TAZ-JNK cascade mediates atheroprotective effect of unidirectional shear flow. Nature. 2016;540(7634):579-582.

21. Nakajima H, et al. Flow-dependent endothelial yap regulation contributes to vessel mainte- nance. Dev Cell. 2017;40(6):523-536.e6.

22. Strasser GA, Kaminker JS, Tessier-Lavigne M. Microarray analysis of retinal endothelial tip cells identifies CXCR4 as a mediator of tip cell morphology and branching. Blood. 2010;115(24):5102-5110.

23. Xin M, et al. Regulation of insulin-like growth factor signaling by Yap governs cardiomyocyte proliferation and embryonic heart size. Sci Signal. 2011;4(196):ra70.

24. Xin M, et al. Hippo pathway effector Yap promotes cardiac regeneration. Proc Natl Acad Sci US A. 2013;110(34):13839-13844.

25. Okabe K, et al. Neurons limit angiogenesis by titrating VEGF in retina. Cell. 2014;159(3):584-596.

26. Heallen T, et al. Hippo pathway inhibits Wnt signaling to restrain cardiomyocyte proliferation and heart size. Science. 2011;332(6028):458-461.

27. Heallen T, et al. Hippo signaling impedes adult heart regeneration. Development. 2013;140(23):4683-4690.

28. Kim M, et al. cAMP/PKA signalling reinforces the LATS-YAP pathway to fully suppress YAP in response to actin cytoskeletal changes. EMBO J. 2013;32(11):1543-1555.

29. Hellström M, et al. Dll4 signalling through Notch1 regulates formation of tip cells during angiogenesis. Nature. 2007;445(7129):776-780.

30. Suchting $S$, et al. The Notch ligand Delta-like 4 negatively regulates endothelial tip cell formation and vessel branching. Proc Natl Acad Sci U S A. 2007;104(9):3225-3230.

31. Wang Y, Rattner A, Zhou Y, Williams J, Smallwood PM, Nathans J. Norrin/Frizzled4 signaling in retinal vascular development and blood brain barrier plasticity. Cell. 2012;151(6):1332-1344.

32. Armulik A, et al. Pericytes regulate the bloodbrain barrier. Nature. 2010;468(7323):557-561.

33. Fan R, Kim NG, Gumbiner BM. Regulation of Hippo pathway by mitogenic growth factors via phosphoinositide 3-kinase and phosphoinositidedependent kinase-1. Proc Natl Acad Sci US A. 2013;110(7):2569-2574.

34. Zhao B, et al. TEAD mediates YAP-dependent gene induction and growth control. Genes Dev. 2008;22(14):1962-1971.

35. Suehiro J, Hamakubo T, Kodama T, Aird WC, Minami T. Vascular endothelial growth factor activation of endothelial cells is mediated by early growth response-3. Blood. 2010;115(12):2520-2532.

36. Potente M, Carmeliet P. The Link Between Angiogenesis and Endothelial Metabolism. Annu Rev Physiol. 2017;79:43-66.

37. Ghesquière B, Wong BW, Kuchnio A, Carmeliet P. Metabolism of stromal and immune cells in health and disease. Nature. 2014;511(7508):167-176.

38. De Bock K, Georgiadou M, Carmeliet P. Role of endothelial cell metabolism in vessel sprouting. Cell Metab. 2013;18(5):634-647.

39. Dang CV. MYC, metabolism, cell growth, and tumorigenesis. Cold Spring Harb Perspect Med. 2013;3(8):a014217.

40. Wilhelm K, et al. FOXO1 couples metabolic activity and growth state in the vascular endothelium. Nature. 2016;529(7585):216-220.

41. Mori M, et al. Hippo signaling regulates microprocessor and links cell-density- dependent miRNA biogenesis to cancer. Cell. 2014;156(5):893-906.

42. Franco CA, et al. SRF selectively controls tip cell invasive behavior in angiogenesis. Development. 2013;140(11):2321-2333.

43. Franco CA, et al. Serum response factor is required for sprouting angiogenesis and vascular integrity. Dev Cell. 2008;15(3):448-461.

44. Weinl C, et al. Endothelial SRF/MRTF ablation causes vascular disease phenotypes in murine retinae. J Clin Invest. 2013;123(5):2193-2206.

45. Olson EN, Nordheim A. Linking actin dynamics and gene transcription to drive cellular motile functions. Nat Rev Mol Cell Biol. 2010;11(5):353-365.

46. Esnault C, et al. Rho-actin signaling to the MRTF coactivators dominates the immediate transcriptional response to serum in fibroblasts. Genes Dev. 2014;28(9):943-958.

47. Kim T, et al. A basal-like breast cancer-specific role for SRF-IL6 in YAP-induced cancer stemness. Nat Commun. 2015;6:10186.

48. Kim T, Hwang D, Lee D, Kim JH, Kim SY, Lim DS. MRTF potentiates TEAD-YAP transcriptional activity causing metastasis. EMBO J 2017;36(4):520-535.

49. Yu OM, Miyamoto S, Brown JH. Myocardin-related transcription factor $A$ and yes-associated protein exert dual control in G protein-coupled receptorand Rhoa-mediated transcriptional regulation and cell proliferation. Mol Cell Biol. 2016;36(1):39-49.

50. Speight P, Kofler M, Szászi K, Kapus A. Context-dependent switch in chemo/mechanotransduction via multilevel crosstalk among cytoskeleton-regulated MRTF and TAZ and TGF $\beta$-regulated Smad3. Nat Commun. 2016;7:11642.

51. del Toro R, et al. Identification and functional analysis of endothelial tip cell-enriched genes. Blood. 2010;116(19):4025-4033.

52. Kim M, et al. Opposing actions of angiopoietin-2 on Tie2 signaling and FOXO1 activation. J Clin Invest. 2016;126(9):3511-3525.

53. Korhonen EA, et al. Tie1 controls angiopoietin function in vascular remodeling and inflammation. J Clin Invest. 2016;126(9):3495-3510.

54. Felcht M, et al. Angiopoietin-2 differentially regulates angiogenesis through TIE2 and integrin signaling. JClin Invest. 2012;122(6):1991-2005.

55. Rocha SF, et al. Esm1 modulates endothelial tip cell behavior and vascular permeability by enhancing VEGF bioavailability. Circ Res. 2014;115(6):581-590.

56. Jaffe AB, Hall A. Rho GTPases: biochemistry and biology. Annu Rev Cell Dev Biol. 2005;21:247-269.

57. Heasman SJ, Ridley AJ. Mammalian Rho GTPases: new insights into their functions from in vivo studies. Nat Rev Mol Cell Biol. 2008;9(9):690-701.

58. Fantin A, et al. NRP1 regulates CDC42 activation to promote filopodia formation in endothelial tip cells. Cell Rep. 2015;11(10):1577-1590.

59. De Smet F, Segura I, De Bock K, Hohensinner PJ, Carmeliet P. Mechanisms of vessel branching: filopodia on endothelial tip cells lead the way. Arterioscler Thromb Vasc Biol. 2009;29(5):639-649.

60. Wakayama Y, Fukuhara S, Ando K, Matsuda M, Mochizuki N. Cdc42 mediates Bmp-induced 
sprouting angiogenesis through Fmnl3-driven assembly of endothelial filopodia in zebrafish. Dev Cell. 2015;32(1):109-122.

61. Barry DM, et al. Cdc42 is required for cytoskeletal support of endothelial cell adhesion during blood vessel formation in mice. Development. 2015;142(17):3058-3070.

62. Vicente-Manzanares M, Ma X, Adelstein RS, Horwitz AR. Non-muscle myosin II takes centre stage in cell adhesion and migration. Nat Rev Mol Cell Biol. 2009;10(11):778-790.

63. Porazinski S, et al. YAP is essential for tissue tension to ensure vertebrate 3D body shape. Nature. 2015;521(7551):217-221.

64. De Bock K, et al. Role of PFKFB3-driven glycolysis in vessel sprouting. Cell. 2013;154(3):651-663.
65. Wang W, et al. AMPK modulates Hippo pathway activity to regulate energy homeostasis. Nat Cell Biol. 2015;17(4):490-499.

66. Schoors $S$, et al. Fatty acid carbon is essential for dNTP synthesis in endothelial cells. Nature. 2015;520(7546):192-197.

67. Shen Z, Stanger BZ. YAP regulates S-phase entry in endothelial cells. PLoS One. 2015;10(1):e0117522.

68. Chow BW, Gu C. The molecular constituents of the blood-brain barrier. Trends Neurosci. 2015;38(10):598-608.

69. Daneman R, Prat A. The blood-brain barrier. Cold Spring Harb Perspect Biol. 2015;7(1):a020412.

70. Obermeier B, Daneman R, Ransohoff RM. Development, maintenance and disruption of the blood- brain barrier. Nat Med. 2013;19(12):1584-1596.

71. Zihni C, Mills C, Matter K, Balda MS. Tight junctions: from simple barriers to multifunctional molecular gates. Nat Rev Mol Cell Biol. 2016;17(9):564-580.

72. Lambert $\mathrm{V}$, et al. Laser-induced choroidal neovascularization model to study age-related macular degeneration in mice. Nat Protoc. 2013;8(11):2197-2211.

73. Lee J, et al. Angiopoietin-1 suppresses choroidal neovascularization and vascular leakage. Invest Ophthalmol Vis Sci. 2014;55(4):2191-2199.

74. Park JR, et al. Imaging laser-induced choroidal neovascularization in the rodent retina using optical coherence tomography angiography. Invest Ophthalmol Vis Sci. 2016;57(9):ОСТ331-ОСТ340. 